\title{
The holographic dual of Rényi relative entropy
}

\author{
Ning Bao, ${ }^{a, b}$ Mudassir Moosa ${ }^{c}$ and Ibrahim Shehzad ${ }^{c}$ \\ ${ }^{a}$ Berkeley Center for Theoretical Physics, \\ Berkeley, CA, 94720, U.S.A. \\ ${ }^{b}$ Computational Science Initiative, Brookhaven National Lab, \\ Upton, NY, 11973, U.S.A. \\ ${ }^{c}$ Department of Physics, Cornell University, \\ Ithaca, NY, 14853, U.S.A. \\ E-mail: ningbao75@gmail.com, mudassir.moosa@cornell.edu, \\ is354@cornell.edu
}

ABSTRACT: The relative entropy is a measure of the distinguishability of two quantum states. A great deal of progress has been made in the study of the relative entropy between an excited state and the vacuum state of a conformal field theory (CFT) reduced to a spherical region. For example, when the excited state is a small perturbation of the vacuum state, the relative entropy is known to have a universal expression for all CFT's [1]. Specifically, the perturbative relative entropy can be written as the symplectic flux of a certain scalar field in an auxiliary AdS-Rindler spacetime [1]. Moreover, if the CFT has a semi-classical holographic dual, the relative entropy is known to be related to conserved charges in the bulk dual spacetime [2]. In this paper, we introduce a one-parameter generalization of the relative entropy which we call refined Rényi relative entropy. We study this quantity in CFT's and find a one-parameter generalization of the aforementioned known results about the relative entropy. We also discuss a new family of positive energy theorems in asymptotically locally AdS spacetimes that arises from the holographic dual of the refined Rényi relative entropy.

KEYwords: AdS-CFT Correspondence, Black Holes, Classical Theories of Gravity, Conformal Field Theory

ARXIV EPRINT: 1904.08433 


\section{Contents}

1 Introduction 1

2 Background $\quad 4$

2.1 Perturbative relative entropy in a general CFT 4

$\begin{array}{lll}2.2 & \text { Quasi-local conserved charges and canonical energy } & 7\end{array}$

2.3 Relative entropy in holographic CFT's 8

3 Preliminaries $\quad 10$

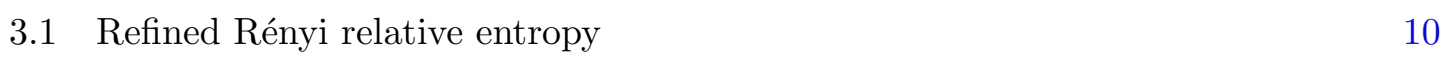

3.1.1 Perturbation of the refined Rényi relative entropy 12

$\begin{array}{lll}3.2 & \text { Sandwiched state in a CFT } & 13\end{array}$

4 Perturbative refined Rényi relative entropy in a general CFT $\quad 15$

$\begin{array}{ll}\text { 4.1 Refined Rényi relative entropy as a symplectic flux } & 18\end{array}$

5 Refined Rényi relative entropy in a holographic CFT 19

$\begin{array}{lll}5.1 \text { Positive energy theorems } & 22\end{array}$

$\begin{array}{ll}5.2 & \text { Relation to the perturbative result } \\ \end{array}$

$\begin{array}{lll}6 & \text { Discussion } & 24\end{array}$

$\begin{array}{ll}\text { A Refined Rényi relative entropy as relative entropy } & 27\end{array}$

$\begin{array}{ll}\text { B Conformal transformation and state } \rho & 28\end{array}$

$\begin{array}{ll}\text { C Refined Rényi relative entropy for thermal states } & 30\end{array}$

\section{Introduction}

The introduction of quantum information theory into quantum gravity in general and the AdS/CFT correspondence in particular has led to a revolution in the latter. Specifically, formulating questions in gravity in terms of entropy measures such as the entanglement entropy and relative entropy has proven to be a very fruitful endeavor.

Relative entropies are a way of quantifying the distinguishability of two quantum states, borne out of quantum information theory. The relative entropy between two states $\rho$ and $\sigma$ is defined as

$$
S_{\text {rel }}(\rho \| \sigma) \equiv \operatorname{tr}(\rho \log \rho)-\operatorname{tr}(\rho \log \sigma) .
$$

This quantity has proven to be very important in the context of quantum gravity, appearing in the proof of the Bekenstein bound $[3,4]$, proofs of the generalized second law $[5,6]$, 
proofs of the Bousso bound [7,8], the proof of the average null energy condition [9], study of the quantum null energy condition [10-12], study of constraints satisfied by the renormalization group trajectories in field theory [13], the question of black hole microstate distinguishability [14], the proof of entanglement wedge reconstruction [15-18], the derivations of bulk constraints such as Einstein equations [19-23], and the recent holographic proofs of the positive energy theorems [2, 24-26].

A significant effort has been made to study relative entropy in the context of conformal field theories (CFT's) on $R^{1, d-1}$, especially when the reference state is taken to be the vacuum state reduced to a spherical region. The relative entropy between an excited state $(\rho)$ and the vacuum state $(\sigma)$ in this setup has been studied using field theory methods in $[1,27,28]$ and using the AdS/CFT correspondence in $[2,19-26,29]$. In the case where the state $\rho$ in eq. (1.1) can be written as small perturbation around the vacuum state $\sigma$, the relative entropy (to lowest order in the perturbation parameter) can be written in terms of the CFT two point functions [1,27], and hence, is completely fixed by conformal symmetry. ${ }^{1}$ This universal perturbative relative entropy for a general CFT was written in [1] as the symplectic flux of a scalar field in an auxiliary AdS-Rindler 'bulk' spacetime

$$
S_{\text {rel }}(\rho \| \sigma)=\int_{\Sigma_{0}} \omega_{\phi}\left(\Phi(r, t, y), £_{\xi} \Phi(r, t, y)\right),
$$

where $\xi$ is related to the timelike killing vector field and $\Sigma_{0}$ is a Cauchy slice in the AdSRindler spacetime; (see section 2.1 for details). On the other hand, when the excited state $\rho$ is not necessarily a perturbative state, the relative entropy between $\rho$ and $\sigma$ depends on the details of the CFT's. The relative entropy in this case was studied for holographic CFT's in [2]. It was argued that the bulk dual of the relative entropy is related to the difference in the conserved charges, $H_{\xi}\left(\mathcal{M}_{\rho}\right)$ and $H_{\xi}\left(\mathcal{M}_{\sigma}\right)$, in the entanglement wedges of $\rho$ and $\sigma$ respectively. That is, [2]

$$
S_{\mathrm{rel}}(\rho \| \sigma)=H_{\xi}\left(\mathcal{M}_{\rho}\right)-H_{\xi}\left(\mathcal{M}_{\sigma}\right) .
$$

The conserved charges $H_{\xi}$ have contributions from both the codimension-2 extremal surface and the asymptotic boundary. We review the concept of conserved charges in section 2.2 and review the derivation of eq. (1.3) in section 2.3 .

Another area of some recent interest has been that of studying the Rényi entropies, $S_{n}(\rho)$, defined by

$$
S_{n}(\rho)=\frac{1}{1-n} \log \operatorname{tr} \rho^{n}
$$

In particular, the holographic dual of a specific derivative of this quantity, called the refined Rényi entropy $\widetilde{S}_{n}(\rho)$, defined by

$$
\widetilde{S}_{n}(\rho)=n^{2} \partial_{n}\left(\frac{n-1}{n} S_{n}(\rho)\right)
$$

was derived in [39], where it was shown that this object is calculated by the minimal area of a cosmic brane with tension given by $T_{n}=\frac{n-1}{4 n G_{N}}$. In the case when $n$ goes to one, this

\footnotetext{
${ }^{1}$ This is true for the perturbative entanglement entropy as well [30-38].
} 
becomes the holographic formula for entanglement entropy [40-43] and corresponds to the tension-less limit of this brane. It is worth noting that while this refined Rényi entropy has a natural holographic dual, the Rényi entropy itself does not yet possess the same.

Since the relative entropy is defined in the context of entanglement entropies, as, for example, is apparent from the fact that entanglement entropy appears explicitly in the form of the relative entropy, it is natural to ask for Rényi generalizations thereof. The problem is that the method for performing this generalization is not unique. Indeed, several different candidates have appeared in the literature, with some of the desirable properties that such a quantity should be expected to possess; see for example [44-48]. In particular, the quantity of the sandwiched Rényi relative entropy defined in these works seems to be the generalization that most preserves the desired properties, in particular the monotonicity of Rényi relative entropy under CPTP maps. This object is defined to be

$$
S_{n}(\rho \| \sigma) \equiv \frac{1}{n-1} \log \operatorname{tr}\left\{\left(\sigma^{\frac{1-n}{2 n}} \rho \sigma^{\frac{1-n}{2 n}}\right)^{n}\right\} .
$$

It is now a natural question to ask whether such a generalization of the relative entropy can be nicely related to a geometric object with nice bulk properties via holography. The goal of this paper is to address this question. Specifically, we consider a refined version of the sandwiched Rényi relative entropy, which we define as

$$
\widetilde{S}_{n}(\rho \| \sigma) \equiv n^{2} \partial_{n}\left(\frac{n-1}{n} S_{n}(\rho \| \sigma)\right)
$$

We call this quantity 'refined' Rényi relative entropy. In particular, we study this quantity when the state $\sigma$ is the vacuum state of an arbitrary CFT reduced to a spherical region and $\rho$ is a slightly excited state. We find that, for integer $n \geq 1$, the perturbative refined Rényi relative entropy, just like the relative entropy, is completely fixed by conformal symmetry and can be written as the symplectic flux of a scalar field in an auxiliary AdSRindler spacetime. We therefore consider our result to be a one-parameter generalization of eq. (1.2). Moreover, we also specialize to the case of holographic CFT's and argue that the holographic dual of the non-perturbative refined Rényi relative entropy, for integer $n \geq 1$, is related to conserved charges in an asymptotically locally AdS spacetime. We consider this to be a one-parameter generalization of eq. (1.3). Finally, we use this holographic formula to prove certain 'positive energy' theorems in the asymptotically locally AdS spacetimes.

It is important to point out here that the Hilbert space of a quantum field theory is not factorizable into subspaces of spatial subregions. Therefore, the 'reduced' density matrices of a spatial subregion are not formally defined objects in quantum field theory. Moreover, local algebras in a quantum field theory are of Type III [49-51], and therefore do not have a notion of trace. Hence, the definition of the relative entropy in eq. (1.1) and sandwiched Rényi relative entropy in eq. (1.6) are only valid for finite dimensional Hilbert spaces. Nevertheless, the relative entropy between a general and a vacuum state on a subregion is still a well-defined object in quantum field theory. This is defined in terms of subalgebras of local operators in the subregion using Tometa-Takesaki modular theory $[52,53]$; see [54] for review. Furthermore, a formal definition of the sandwiched 
Rényi relative entropy was recently discussed in [55]. Since, the sandwiched Rényi relative entropy is a well-defined quantity in a quantum field theory, we deduce from eq. (1.7) that the refined Rényi relative entropy is also a well-defined object in quantum field theory.

The rest of this paper in organized as follows. We start with a review of known results about relative entropy in CFT's, such as eq. (1.2) and eq. (1.3), and a review of conserved charges using covariant phase space methods in section 2 . We introduce refined Rényi relative entropy in section 3.1 and derive some properties of this quantity. In particular, we show that this quantity can be written as the relative entropy of an $n$ dependent state, which we call the 'sandwiched' state. We use this observation to derive a general perturbative formula for the refined Rényi relative entropy in section 3.1.1. We then show in section 3.2 that for a particular family of states, the sandwiched state can be prepared by a Euclidean path integral. Using these results, we study the refined Rényi relative entropy in section 4 when the reference state is the vacuum of a CFT reduced to a spherical region and the other state is a small perturbation thereof. We show in section 4.1 that the refined Rényi relative entropy in this case can be written as the symplectic flux of a scalar field through a Cauchy slice of the AdS-Rindler spacetime, thus providing a one-parameter generalization of eq. (1.2). We then specialize to CFT's with semi-classical holographic duals in section 5. We argue that the holographic dual of the refined Rényi relative entropy between an excited state and a vacuum state reduced to a spherical region is related to conserved charges in the bulk dual of the sandwiched state. We therefore consider our result to be a one-parameter generalization of eq. (1.3). We use this result in section 5.1 to prove certain 'positive-energy' theorems in asymptotically locally AdS spacetimes. Finally, we end with a summary of our results and some possible future directions in section 6 .

\section{Background}

Our goal in this paper is to present a one-parameter generalization of the known results about the relative entropy in CFT's, particularly eq. (1.2) and eq. (1.3). We dedicate this section to a brief review of these known results as well as a brief review of the concept of the conserved charges derived via covariant phase space methods.

\subsection{Perturbative relative entropy in a general CFT}

As pointed out earlier, relative entropy has been studied in detail between states of a conformal field theory $(\mathrm{CFT})$ in $R^{1, d-1}$ especially when the reference state is taken to be the vacuum state reduced to a spherical region. The simplification that occurs when dealing with the CFT vacuum reduced to a spherical region, $B$, is that the vacuum reduced state, $\sigma$, has a universal form [56]. In particular, the modular Hamiltonian of the reduced vacuum state, defined as $K_{\sigma} \equiv-\log \sigma$, is given by [56]

$$
K_{\sigma}=\int_{\mathcal{S}} d \Sigma^{b} \hat{\xi}^{a} T_{a b}
$$

where $\mathcal{S}$ is any achronal slice within the domain of dependence of the spherical region $B$, $\mathcal{D}(B)$, such that $\partial \mathcal{S}=\partial B$. Moreover, the vector $\hat{\xi}$ is the generator of a (modular) flow 
in $\mathcal{D}(B)$. In particular, the vector field $\hat{\xi}$ (in Cartesian coordinates $x^{\mu}=\left\{x^{0}, x^{i}\right\}$ ) for a spherical region of radius $R$ at $x^{0}=0$ and centered at the origin is

$$
\hat{\xi}=\frac{\pi\left(R^{2}-\left(x^{0}\right)^{2}-\vec{x}^{2}\right)}{R} \partial_{0}-\frac{2 \pi x^{0} x^{i}}{R} \partial_{i} .
$$

For holographic CFT's on $R^{1, d-1}$, the vacuum state is dual to the Poincare patch of the $(d+1)$-dimensional vacuum AdS spacetime, which is described by

$$
d s^{2}=\frac{\ell^{2}}{z^{2}}\left(d z^{2}+\eta_{\mu \nu} d x^{\mu} d x^{\nu}\right) .
$$

where $z=0$ is the boundary of the AdS spacetime and $\ell$ is the AdS length scale. Moreover, the bulk region dual to the vacuum state reduced to a subregion is the corresponding entanglement wedge in Poincare AdS. The entanglement wedge corresponding to a spherical region $B$ of radius $R, \mathcal{M}_{\sigma}$, is the domain of dependence of an achronal surface, $\Sigma_{0}$, such that $\partial \Sigma_{0}=B \cup \tilde{B}_{\sigma}$, where

$$
\tilde{B}_{\sigma}: \quad x^{0}=0 \quad \text { and } \quad z^{2}+\vec{x}^{2}=R^{2} .
$$

The vector field, $\xi$, that generates the (modular) flow in the entanglement wedge $\mathcal{M}_{\sigma}$ is

$$
\xi^{z}=-\frac{2 \pi x^{0} z}{R} \quad \xi^{0}=\frac{\pi\left(R^{2}-z^{2}-\left(x^{0}\right)^{2}-\vec{x}^{2}\right)}{R} \quad \xi^{i}=\hat{\xi}^{i} .
$$

It is easy to check that $\xi$ is a killing vector field of the Poincare AdS spacetime and that it satisfies the following boundary conditions

$$
\left.\xi\right|_{B}=\left.\hat{\xi} \quad \xi\right|_{\tilde{B}_{\sigma}}=0
$$

The entanglement wedge $\mathcal{M}_{\sigma}$ can be mapped, by a coordinate transformation, to the AdS-Rindler spacetime [56], which is described by

$$
d s^{2}=-\left(\frac{r^{2}}{R^{2}}-1\right) d t^{2}+\left(\frac{r^{2}}{R^{2}}-1\right)^{-1} d r^{2}+r^{2} d s_{\mathbb{H}^{d-1}}^{2},
$$

where $d s_{\mathbb{H}^{d-1}}^{2}$ is the metric on the $(d-1)$-dimensional unit hyperbolic space. In this coordinate system, the extremal surface $\tilde{B}_{\sigma}$ is given by $r=R$ for any finite $t$, which is the bifurcation surface of the AdS-Rindler spacetime. Moreover, $\xi$ is related to the timetranslation vector field by

$$
\xi=-2 \pi R \partial_{t}
$$

One can now check that the vector field $\xi$ satisfies

$$
\left.\left(\nabla^{a} \xi^{b}-\nabla^{b} \xi^{a}\right)\right|_{\tilde{B}_{\sigma}}=4 \pi n^{a b}
$$

where $n^{a b} \equiv n_{1}^{a} n_{2}^{b}-n_{2}^{a} n_{1}^{b}$, where $n_{1}=\partial_{r}$ and $n_{2}=\partial_{t}$ are normal vectors to the extremal surface $\tilde{B}_{\sigma}$. 
Now let us consider an excited state of a CFT prepared by a path integral with the insertion of a smeared operator. We denote this state reduced to a spherical region $B$ by $\rho$. For the case when the excited state $\rho$ is a perturbation of $\sigma$, the relative entropy between these states for holographic CFT's was studied using the AdS/CFT correspondence in [26]. This work was generalized in [1] for all CFT's, irrespective of whether the CFT has a holographic dual or not. It was shown in [1] that the perturbative relative entropy can be written as the symplectic flux of a scalar field through a Cauchy slice of the AdS-Rindler spacetime. More precisely,

$$
S_{\text {rel }}(\rho \| \sigma)=\int_{\Sigma_{0}} \omega_{\phi}\left(\Phi(r, t, y), £_{\xi} \Phi(r, t, y)\right) .
$$

The mass of the scalar field in eq. (2.10) is fixed by the conformal dimension of the operator used to prepare the perturbed state $\rho$ whereas the boundary condition of the scalar field is fixed by the smearing function of that operator. If the CFT under consideration were holographic, this scalar field would have been the holographic dual of the operator used to prepare the state $\rho$. However, it should be noted that the AdS/CFT correspondence was not assumed in the analysis of [1] and hence, the result in eq. (2.10) is valid for all CFT's. Therefore, the scalar field in eq. (2.10) and the AdS-Rindler spacetime should be thought as auxiliary tools used to write the perturbed relative entropy geometrically.

Recently, a similar perturbative calculation was performed in [57] for the Rényi relative divergence which is defined as

$$
D_{n}(\rho \| \sigma) \equiv \operatorname{tr}\left(\sigma^{1-n} \rho^{n}\right) .
$$

In particular, it was found that at the lowest order in the perturbation parameter, the quantity defined as

$$
\tilde{D}_{n}(\rho \| \sigma) \equiv D_{-n}(\rho \| \sigma)-D_{n}(\rho \| \sigma),
$$

and its derivative, $\partial_{n} \tilde{D}_{n}(\rho \| \sigma)$, can also be written as the symplectic flux of the scalar field as in eq. (1.2). The perturbative Rényi relative divergence was also studied in [58], where the Rényi relative divergence between two thermal states was written as a Euclidean path integral. This path integral was then computed using the AdS/CFT correspondence.

The sandwiched Rényi relative entropy and the Rényi relative divergence are both special cases of $n-z$ Rényi relative divergence which is defined as

$$
D_{n, z}(\rho \| \sigma) \equiv \frac{1}{n-1} \log \operatorname{tr}\left\{\left(\sigma^{\frac{1-n}{2 z}} \rho^{\frac{n}{z}} \sigma^{\frac{1-n}{2 z}}\right)^{z}\right\}
$$

This quantity was studied for two perturbatively nearby states in [59]. Indeed, it was found that at the lowest order in the perturbation parameter, this quantity can also be written as the symplectic flux of the scalar field as in eq. (1.2). However, the AdS/CFT correspondence was assumed in [59] and hence this analysis is only valid for holographic CFT's.

This concludes our review of the known perturbative results regarding relative entropy and various associated Rényi quantities. Before moving on to a review of the derivation of eq. (1.3) presented in [2], we review the notion of conserved charges and canonical energy in the following subsection. 


\subsection{Quasi-local conserved charges and canonical energy}

In this subsection, we switch from quantum information to gravity temporarily and talk about the concepts of conserved charges and canonical energy in the general framework of covariant phase space methods. We restrict ourselves to a review of these quantities only to the extent that will be relevant for our analysis in this paper. The reader is referred to canonical references on this topic [60-63] for full details.

Given a field theory in $(d+1)$ dimensions, any variation of the Lagrangian is given by

$$
\delta L \equiv E(g) \delta g+d \theta(g, \delta g),
$$

where we use $g, \delta g$ as collective labels to represent the metric and matter fields and their variations respectively. In eq. (2.14), $E(g)=0$ denotes the equations of motion and the $d$-form $\theta(g, \delta g)$ is called the symplectic potential. From the symplectic potential, one can define the symplectic current, $\omega\left(g, \delta_{1} g, \delta_{2} g\right)$, as

$$
\omega\left(g, \delta_{1} g, \delta_{2} g\right) \equiv \delta_{1} \theta\left(g, \delta_{2} g\right)-\delta_{2} \theta\left(g, \delta_{1} g\right),
$$

which is a $d$-form and is antisymmetric and bilinear in two field perturbations.

Given $\Sigma$, a subspace of a codimension- 1 hypersurface and $\eta$, a vector field on $\Sigma$, the perturbed Hamiltonian conjugate to $\eta$ is given by [60-63]

$$
\delta H_{\eta} \equiv \int_{\Sigma} \omega\left(g, \delta g, £_{\eta} g\right) .
$$

Using eq. (2.14) and assuming equations of motions, $E(g)=0$, this can be written as

$$
\delta H_{\eta}=\int_{\Sigma} \delta\left(\theta\left(£_{\eta} g\right)-\eta \cdot L\right)-\int_{\partial \Sigma} \eta \cdot \theta(\delta g)
$$

where $\partial \Sigma$ denotes the boundary of $\Sigma$. It is worth pointing out that the existence of the Hamiltonian, $H_{\eta}$, is a non-trivial issue. In particular, it exists if and only if the boundary term is a total variation which is to say that the quantity appearing on the right hand side of eq. (2.17) is integrable. This is guaranteed if the following condition holds [63]

$$
\int_{\partial \Sigma} \eta \cdot\left[\delta_{1} \theta\left(\delta_{2} g\right)-\delta_{2} \theta\left(\delta_{1} g\right)\right]=0,
$$

for arbitrary perturbations $\delta_{1}$ and $\delta_{2}$. When this condition holds, there exists a $d$-form $K(g)$ such that [2]

$$
\delta(\eta \cdot K(g)) \equiv \eta \cdot \theta(\delta g) \quad \text { on } \partial \Sigma .
$$

Assuming the condition in eq. (2.18) is satisfied, the Hamiltonian conjugate to $\eta$ can be written as

$$
H_{\eta}=\int_{\Sigma} J_{\eta}-\int_{\partial \Sigma} \eta \cdot K
$$

where

$$
J_{\eta} \equiv \theta\left(£_{\eta} g\right)-\eta \cdot L
$$


Note that the Hamiltonian, $H_{\eta}$, in eq. (2.20) can be written purely as a boundary integral. This is because $J_{\eta}$ can be written, using equations of motion, as

$$
J_{\eta}=d Q_{\eta}
$$

where $Q_{\eta}$ is a $(d-1)$-form and is called the Noether charge. Therefore, the Hamiltonian can be written as an integral over $\partial \Sigma$,

$$
H_{\eta}=\int_{\partial \Sigma}\left(Q_{\eta}-\eta \cdot K\right)
$$

This 'quasi-local' expression implies that the Hamiltonian only depends on the details of the vector field $\eta$ near the boundary $\partial \Sigma$. Moreover, this implies that the Hamiltonian is the same for any two codimension- 1 surfaces $\Sigma_{1}$ and $\Sigma_{2}$ if they have a common boundary, i.e. $\partial \Sigma_{1}=\partial \Sigma_{2}$. In this sense, the Hamiltonian is conserved and hence we will use it interchangeably with conserved charge in this paper.

Now let us suppose that the metric and matter fields have a perturbative expansion given by

$$
g=g^{(0)}+\epsilon g^{(1)}+O\left(\epsilon^{2}\right),
$$

where $\epsilon \ll 1$ is a perturbation parameter. Let us further assume that the unperturbed metric and matter fields are invariant under the diffeomorphism generated by the vector field $\eta$. That is, $£_{\eta} g^{(0)}=0$. Using eq. (2.16), we deduce that the derivative of the Hamiltonian, $H_{\eta}$, is given by

$$
\frac{d}{d \epsilon} H_{\eta}=\int_{\Sigma} \omega\left(g, \frac{d}{d \epsilon} g, £_{\eta} g\right)
$$

At linear order in $\epsilon$, the Hamiltonian, $H_{\eta}$, is

$$
\left.H_{\eta}^{(1)} \equiv \frac{d H_{\eta}}{d \epsilon}\right|_{\epsilon=0}=\int_{\Sigma} \omega\left(g^{(0)}, g^{(1)}, £_{\eta} g^{(0)}\right) .
$$

Since we have assumed $£_{\eta} g^{(0)}=0$, we get that $H_{\eta}^{(1)}=0$. Similarly, the Hamiltonian, $H_{\eta}$, at quadratic order in $\epsilon$ is given by

$$
\left.H_{\eta}^{(2)} \equiv \frac{d^{2} H_{\eta}}{d \epsilon^{2}}\right|_{\epsilon=0}=\int_{\Sigma} \omega\left(g^{(0)}, g^{(1)}, £_{\eta} g^{(1)}\right) .
$$

The quantity on the right hand side, for the case when $\eta$ is a timelike killing vector field of the unperturbed metric, is called the canonical energy [64].

This concludes our review of the concepts of conserved charges and canonical energy. In the next subsection, we review the derivation of eq. (1.3) originally presented in [2].

\subsection{Relative entropy in holographic CFT's}

Now we consider a CFT with a holographic dual. Like in section 2.1, we denote an excited state and a vacuum state reduced to a spherical region by $\rho$ and $\sigma$ respectively. However, we 
no longer assume that the state $\rho$ is a perturbative state around the vacuum state $\sigma$. The relative entropy between these states was studied in [2] using the AdS/CFT correspondence. It was found that the holographic dual of the relative entropy between these states is related to certain conserved charges in the entanglement wedge corresponding to $\rho, \mathcal{M}_{\rho}$, and in the entanglement wedge corresponding to $\sigma, \mathcal{M}_{\sigma}$. Here, we review the argument of [2].

Recall that the relative entropy in eq. (1.1) can be written as

$$
S_{\mathrm{rel}}(\rho \| \sigma)=S(\sigma)-S(\rho)+\left\langle K_{\sigma}\right\rangle_{\rho}-\left\langle K_{\sigma}\right\rangle_{\sigma}
$$

where $S(\rho)$ and $S(\sigma)$ are the von Neumann entropies of the states $\rho$ and $\sigma$ whereas $\left\langle K_{\sigma}\right\rangle_{\rho}$ and $\left\langle K_{\sigma}\right\rangle_{\sigma}$ are the expectation values of $K_{\sigma}$ in the states $\rho$ and $\sigma$ respectively.

For holographic CFT's, we already know the bulk duals of $S(\sigma)$ and of $S(\rho)$. In particular, these quantities are given by $[40,41]$

$$
\begin{aligned}
& S(\sigma)=\frac{\operatorname{Area}\left(\tilde{B}_{\sigma}\right)}{4 G_{N}}, \\
& S(\rho)=\frac{\operatorname{Area}\left(\tilde{B}_{\rho}\right)}{4 G_{N}},
\end{aligned}
$$

where $\tilde{B}_{\sigma}$ and $\tilde{B}_{\rho}$ are the boundary anchored codimension-2 extremal surface corresponding to the states $\sigma$ and $\rho$ respectively. Now using the form of $K_{\sigma}$ from eq. (2.1) and using eq. (2.29) and eq. (2.30), we write the relative entropy in eq. (2.28) as

$$
S_{\text {rel }}(\rho \| \sigma)=\frac{\operatorname{Area}\left(\tilde{B}_{\sigma}\right)}{4 G_{N}}-\frac{\operatorname{Area}\left(\tilde{B}_{\rho}\right)}{4 G_{N}}+\int_{\mathcal{S}} d \Sigma^{b} \hat{\xi}^{a}\left\langle T_{a b}\right\rangle_{\rho}-\int_{\mathcal{S}} d \Sigma^{b} \hat{\xi}^{a}\left\langle T_{a b}\right\rangle_{\sigma} .
$$

As discussed in section 2.1, $\tilde{B}_{\sigma}$ is the bifurcation surface of the AdS-Rindler spacetime. Hence, the area of this surface can be written as a surface integral of the Noether charge conjugate to $\xi[60,61]$. That is,

$$
\frac{\operatorname{Area}\left(\tilde{B}_{\sigma}\right)}{4 G_{N}}=\int_{\tilde{B}_{\sigma}} Q_{\xi}\left(\mathcal{M}_{\sigma}\right)
$$

where $\xi$ is the killing vector field in eq. (2.5). Moreover, since $\xi$ vanishes at $\tilde{B}_{\sigma}$ as stated in eq. (2.6), we can write eq. (2.32) as

$$
\frac{\operatorname{Area}\left(\tilde{B}_{\sigma}\right)}{4 G_{N}}=\int_{\tilde{B}_{\sigma}}\left(Q_{\xi}\left(\mathcal{M}_{\sigma}\right)-\xi \cdot K\left(\mathcal{M}_{\sigma}\right)\right) .
$$

In general, there is no killing vector field in the bulk dual of an excited CFT state. Therefore, it does not trivially follow that the area of $\tilde{B}_{\rho}$ can also be written as in eq. (2.33). However, it was argued in [2] that one can always find a vector field (which we also denote by $\xi$ ) that vanishes on $\tilde{B}_{\rho}$ and that satisfies eq. (2.9) near $\tilde{B}_{\rho}$. With these conditions on $\xi$, it was shown in [2] that we can write

$$
\frac{\operatorname{Area}\left(\tilde{B}_{\rho}\right)}{4 G_{N}}=\int_{\tilde{B}_{\rho}}\left(Q_{\xi}\left(\mathcal{M}_{\rho}\right)-\xi \cdot K\left(\mathcal{M}_{\rho}\right)\right)
$$


If the state $\rho$ is a perturbative state around the vacuum, then it was shown in [20] that

$$
\begin{aligned}
\int_{B}\left(Q_{\xi}\left(\mathcal{M}_{\rho}\right)-\xi \cdot K\left(\mathcal{M}_{\rho}\right)\right)- & \int_{B}\left(Q_{\xi}\left(\mathcal{M}_{\sigma}\right)-\xi \cdot K\left(\mathcal{M}_{\sigma}\right)\right) \\
= & \int_{\mathcal{S}} d \Sigma^{b} \hat{\xi}^{a}\left\langle T_{a b}\right\rangle_{\rho}-\int_{\mathcal{S}} d \Sigma^{b} \hat{\xi}^{a}\left\langle T_{a b}\right\rangle_{\sigma}
\end{aligned}
$$

where the integral is performed over a spherical boundary region $B$. It was then argued in [2] that this expression holds even if $\rho$ is not a perturbative state.

Now using eq. (2.33), eq. (2.34), and eq. (2.35), we write eq. (2.31) as

$$
\begin{aligned}
S_{\mathrm{rel}}(\rho \| \sigma)= & \int_{B}\left(Q_{\xi}\left(\mathcal{M}_{\rho}\right)-\xi \cdot K\left(\mathcal{M}_{\rho}\right)\right)-\int_{\tilde{B}_{\rho}}\left(Q_{\xi}\left(\mathcal{M}_{\rho}\right)-\xi \cdot K\left(\mathcal{M}_{\rho}\right)\right) \\
& -\int_{B}\left(Q_{\xi}\left(\mathcal{M}_{\sigma}\right)-\xi \cdot K\left(\mathcal{M}_{\sigma}\right)\right)+\int_{\tilde{B}_{\sigma}}\left(Q_{\xi}\left(\mathcal{M}_{\sigma}\right)-\xi \cdot K\left(\mathcal{M}_{\sigma}\right)\right) .
\end{aligned}
$$

Note that $B \cup \tilde{B}_{\sigma}$ and $B \cup \tilde{B}_{\rho}$ are the boundaries of achronal slices on $\mathcal{M}_{\sigma}$ and $\mathcal{M}_{\rho}$ respectively. Now using eq. (2.23), we write the relative entropy in eq. (2.36) as

$$
S_{\mathrm{rel}}(\rho \| \sigma)=H_{\xi}\left(\mathcal{M}_{\rho}\right)-H_{\xi}\left(\mathcal{M}_{\sigma}\right)
$$

In this equation, $H_{\xi}\left(\mathcal{M}_{\sigma}\right)$ is the conserved charge corresponding to the diffeomorphism generated in $\mathcal{M}_{\sigma}$ by the killing vector field $\xi$ given in eq. (2.5). On the other hand, $H_{\xi}\left(\mathcal{M}_{\rho}\right)$ is the conserved charge corresponding to the diffeomorphism generated by any vector field in $\mathcal{M}_{\rho}$ that behaves like the vector field $\xi$ near the boundary of $\mathcal{M}_{\rho}$. That is, it is a killing vector field near the asymptotic boundary and it satisfies boundary conditions similar to eq. (2.6) and eq. (2.9).

This finishes our review of the known results about relative entropy in the context of CFT's and holography, especially eq. (1.2) and eq. (1.3). Our goal in this paper is to show that similar results are true for the refined Rényi relative entropy defined in eq. (1.7). Before we do this, we discuss the refined Rényi relative entropy and derive some of its properties in the next section.

\section{Preliminaries}

\subsection{Refined Rényi relative entropy}

We start by reviewing two quantities in quantum information theory that are particularly relevant to this paper, namely relative entropy and Rényi relative entropy. In doing so, we will show that for particular choices of states, relative entropy and a quantity related to Rényi relative entropy, the refined Rényi relative entropy, are actually equal to each other. This equality will be central to our analysis in the subsequent sections of this paper.

Recall that the relative entropy between two states $\rho$ and $\sigma$ is defined as

$$
S_{\text {rel }}(\rho \| \sigma) \equiv \operatorname{tr}(\rho \log \rho)-\operatorname{tr}(\rho \log \sigma)
$$


when $\operatorname{supp}(\rho) \subseteq \operatorname{supp}(\sigma)$ (otherwise, the relative entropy is taken to be $\infty$ ). The relative entropy satisfies a data-processing inequality which implies that the relative entropy decreases under a completely positive and trace preserving map (CPTP) [65]. That is, under a CPTP map $\mathcal{E}$,

$$
S_{\text {rel }}(\mathcal{E}(\rho) \| \mathcal{E}(\sigma)) \leq S_{\text {rel }}(\rho \| \sigma) .
$$

Now consider two reduced density states of a subsystem $A, \rho_{A}$ and $\sigma_{A}$. For any smaller subsystem $B \subset A$, eq. (3.2) implies

$$
S_{\text {rel }}\left(\rho_{B} \| \sigma_{B}\right) \leq S_{\text {rel }}\left(\rho_{A} \| \sigma_{A}\right) .
$$

In other words, it is more difficult to distinguish two states when some potentially distinguishing information is traced out.

The one-parameter generalization of the relative entropy that also satisfies a dataprocessing inequality is the 'sandwiched' Rényi relative entropy, which is defined as [44-48]

$$
S_{n}(\rho \| \sigma) \equiv \frac{1}{n-1} \log \operatorname{tr}\left\{\left(\sigma^{\frac{1-n}{2 n}} \rho \sigma^{\frac{1-n}{2 n}}\right)^{n}\right\},
$$

when $\operatorname{supp}(\rho) \subseteq \operatorname{supp}(\sigma)$. This quantity also monotonically increases with Rényi parameter $n[45,47]$. That is,

$$
\partial_{n} S_{n}(\rho \| \sigma) \geq 0
$$

In this work, we introduce a closely related quantity, refined Rényi relative entropy, which is defined as

$$
\widetilde{S}_{n}(\rho \| \sigma) \equiv n^{2} \partial_{n}\left(\frac{n-1}{n} S_{n}(\rho \| \sigma)\right) .
$$

Just like the sandwiched relative entropy, this quantity approaches the relative entropy between states $\rho$ and $\sigma$ in the limit $n \rightarrow 1$. That is,

$$
\lim _{n \rightarrow 1} \widetilde{S}_{n}(\rho \| \sigma)=S_{\text {rel }}(\rho \| \sigma) .
$$

Note that we can write eq. (3.6) as

$$
\widetilde{S}_{n}(\rho \| \sigma)=S_{n}(\rho \| \sigma)+n(n-1) \partial_{n} S_{n}(\rho \| \sigma) .
$$

Now using the monotonicity of sandwiched Rényi relative entropy, eq. (3.5), we find the following hierarchical structure between the different relative entropies

$$
\begin{array}{ll}
\widetilde{S}_{n}(\rho \| \sigma) \geq S_{n}(\rho \| \sigma) \geq S_{\text {rel }}(\rho \| \sigma) & \text { for } n \geq 1 \\
\widetilde{S}_{n}(\rho \| \sigma) \leq S_{n}(\rho \| \sigma) \leq S_{\text {rel }}(\rho \| \sigma) & \text { for } n \leq 1 .
\end{array}
$$

As we will see in section 5.1, applying the inequality in eq. (3.9) in the context of the AdS/CFT correspondence leads to interesting energy conditions in asymptotically AdS spacetimes. 
A useful property of the refined Rényi relative entropy is that it can be written as the relative entropy of a related state. More precisely, we have

$$
\widetilde{S}_{n}(\rho \| \sigma)=S_{\text {rel }}\left(\rho_{(n)} \| \sigma\right),
$$

where we have introduced a 'sandwiched' state, $\rho_{(n)}$, which is defined as

$$
\rho_{(n)} \equiv \frac{\left(\sigma^{\frac{1-n}{2 n}} \rho \sigma^{\frac{1-n}{2 n}}\right)^{n}}{\operatorname{tr}\left(\sigma^{\frac{1-n}{2 n}} \rho \sigma^{\frac{1-n}{2 n}}\right)^{n}} .
$$

We relegate the derivation of eq. (3.11) to appendix A and discuss the consequences of this identity in the following. First, the fact that the relative entropy is non-negative guarantees that the refined Rényi relative entropy is also non-negative.

$$
\widetilde{S}_{n}(\rho \| \sigma) \geq 0 .
$$

Secondly, the identity in eq. (3.11) allows us to study the perturbative expansion of the refined Rényi relative entropy which we discuss in detail in the next subsection.

\subsubsection{Perturbation of the refined Rényi relative entropy}

In this section, our goal is to study the refined Rényi relative entropy when the state $\rho$ is perturbatively expanded around an arbitrary state $\sigma$. More precisely, we take state $\rho$ to be given by

$$
\rho=\sigma+\epsilon \rho^{(1)}+O\left(\epsilon^{2}\right)
$$

where $\epsilon \ll 1$ is a perturbation parameter. The perturbation of the relative entropy has been studied in the literature $[1,26,27]$. Since the relative entropy is non-negative, it means that the relative entropy between states $\rho$ and $\sigma$ vanishes at linear order in $\epsilon$. This implies that the change in entanglement entropy is equal to the change in 'modular' energy, which is usually called the first law of entanglement [29]. Therefore, to lowest order in $\epsilon$, the relative entropy between states $\rho$ and $\sigma$ is of the form

$$
S_{\mathrm{rel}}(\rho \| \sigma)=\frac{\epsilon^{2}}{2} S_{\mathrm{rel}}^{(2)}(\rho \| \sigma)+O\left(\epsilon^{3}\right)
$$

where [1]

$$
S_{\text {rel }}^{(2)}(\rho \| \sigma)=-\int_{-\infty}^{\infty} \frac{d s}{4 \sinh ^{2}\left(\frac{s+i \delta}{2}\right)} \operatorname{tr}\left(\sigma^{-1-\frac{i s}{2 \pi}} \rho^{(1)} \sigma^{\frac{i s}{2 \pi}} \rho^{(1)}\right) .
$$

Since the refined Rényi relative entropy is related to the relative entropy according to eq. (3.11), we can deduce the second-order contribution to the refined Rényi relative entropy using eq. (3.16). To do this, we first need to find the perturbative expansion of the sandwiched state, $\rho_{(n)}$. By inserting the expansion of $\rho$ from eq. (3.14) in eq. (3.12), we find that the sandwiched state for integer $n \geq 1$ at linear order in $\epsilon$ is given by

$$
\rho_{(n)}=\sigma+\epsilon \sum_{k=0}^{n-1} \sigma^{\frac{1-n+2 k}{2 n}} \rho^{(1)} \sigma^{\frac{n-1-2 k}{2 n}}+O\left(\epsilon^{2}\right) .
$$


Now using eq. (3.11) and eq. (3.16), we deduce that the refined Rényi relative entropy at the lowest order in $\epsilon$ is

$$
\widetilde{S}_{n}(\rho \| \sigma)=\frac{\epsilon^{2}}{2} \widetilde{S}_{n}^{(2)}(\rho \| \sigma)+O\left(\epsilon^{3}\right)
$$

where

$$
\widetilde{S}_{n}^{(2)}(\rho \| \sigma)=-\sum_{k=0}^{n-1} \sum_{j=0}^{n-1} \int_{-\infty}^{\infty} \frac{d s}{4 \sinh ^{2}\left(\frac{s+i \delta}{2}\right)} \operatorname{tr}\left(\sigma^{-1} \sigma^{-\frac{i s}{2 \pi}+\frac{(k-j)}{n}} \rho^{(1)} \sigma^{\frac{i s}{2 \pi}-\frac{(k-j)}{n}} \rho^{(1)}\right) .
$$

This general formula for the perturbation of the refined Rényi relative entropy for integer $n \geq 1$ is the main result of this section. ${ }^{2}$ We will use this result in section 4 to relate the perturbative refined Rényi relative entropy to the symplectic flux of a scalar field through a Cauchy slice of the AdS-Rindler wedge.

\subsection{Sandwiched state in a CFT}

In this paper, we are interested in studying the refined Rényi relative entropy between states of a conformal field theory $(\mathrm{CFT})$ on $R^{1, d-1}$ though our results are valid for CFT's on $R \times S^{d-1}$ as well. In particular, our approach will be to use the relation between the refined Rényi relative entropy and the relative entropy given in eq. (3.11). To use this relation, we first need to construct the sandwiched state, $\rho_{(n)}$, for given states $\rho$ and $\sigma$. In this section, we show that for a family of states $\rho$ and $\sigma$, the sandwiched state $\rho_{(n)}$ can be prepared (up to a unitary transformation) by a Euclidean path integral over $\mathcal{H}^{\prime}=S^{1} \times \mathbb{H}^{d-1}$.

Let us take the state $\sigma$ to be the vacuum state of the CFT, $|\Omega\rangle$, reduced to a spherical region, $B$, of radius $R$. The domain of dependence of $B$, which we denote by $\mathcal{D}(B)$, can be mapped to $\mathcal{H}=R \times \mathbb{H}^{d-1}$ by a conformal transformation [56]. Under this transformation, the state $\sigma$ maps to a thermal state, $\tilde{\sigma}$, of temperature $T=1 /(2 \pi R)$ on the hyperbolic space of radius $R$ [56]. Therefore, the state $\sigma$ is related to $\tilde{\sigma}$ by a unitary transformation. If we denote the Hamiltonian on the hyperbolic space by $H$, then the state $\sigma$ is given by

$$
U \sigma U^{\dagger}=\tilde{\sigma} \equiv \frac{e^{-2 \pi R H}}{Z_{R}},
$$

where $Z_{R}=\operatorname{tr} e^{-2 \pi R H}$ is the thermal partition function on the hyperbolic space and $U$ is the unitary transformation that maps states from $B$ to hyperbolic space.

Following [55], We now consider a state $|\Psi\rangle \equiv \mathcal{N} \Psi|\Omega\rangle$, where $\Psi$ is defined by the smearing of a local operator $\Psi(x)$ in a small neighborhood around the point $x$ and $\mathcal{N}$ is the normalization constant. We take state $\rho$ to be the state $|\Psi\rangle$ reduced to region $B$. The state $\rho$ can be represented by a Euclidean path integral on $R^{d}$ with open cuts just above and below the region $B$ and with insertions of operators $\Psi$ in the lower and in the upper half plane. ${ }^{3}$

\footnotetext{
${ }^{2}$ It may be possible to analytically continue eq. (3.19) to non-integer $n$ by converting the sum into a contour integral as done in [27]. We thank Tom Hartman for pointing this out.

${ }^{3}$ For simplicity, we take $\Psi$ to be a Hermitian operator.
} 


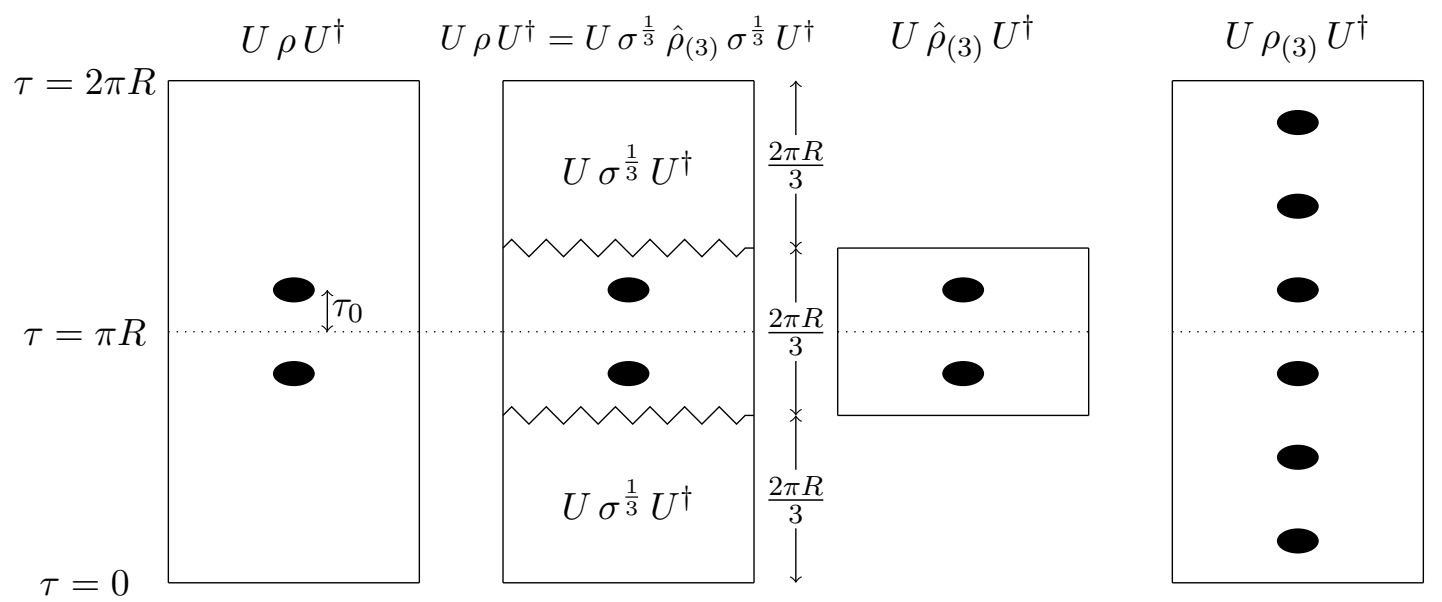

Figure 1. The pictorial representation of the construction of the states $\rho$ and $\rho_{(n)}$ using a Euclidean path integral over $\mathcal{H}^{\prime}=S^{1} \times \mathbb{H}^{d-1}$. The vertical direction denotes the direction along the $S^{1}$ whereas the horizontal direction denotes the radial direction of $\mathbb{H}^{d-1}$ and we have suppressed the transverse $d-2$ dimensions. (This figure is inspired by figure 1 of [2].)

Just like the state $\sigma$, the state $\rho$ is also related to the state $\tilde{\rho}$ on the hyperbolic space by a unitary transformation. To find the state $\tilde{\rho}$, we use the conformal transformation from $R^{d}$ to $\mathcal{H}^{\prime}=S^{1} \times \mathbb{H}^{d-1}$ where the radii of $S^{1}$ and $\mathbb{H}^{d-1}$ are equal to $R$. Note that this is simply the Euclidean version of the conformal transformation that maps $\mathcal{D}(B)$ to $\mathcal{H}$. Under this conformal transformation, the aforementioned path integral representation of the state $\rho$ maps to a path integral representation of a density matrix on $\mathcal{H}^{\prime}$. We perform this analysis in appendix B and find that the matrix elements of $\rho$ and $\tilde{\rho}$, up to a normalization constant, can be written as

$$
\begin{aligned}
\left\langle\tilde{\phi}_{-}\left|U \rho U^{\dagger}\right| \tilde{\phi}_{+}\right\rangle & =\left\langle\tilde{\phi}_{-}|\tilde{\rho}| \tilde{\phi}_{+}\right\rangle \\
& \sim \int_{\Phi(\tau=0)=\tilde{\phi}_{+}}^{\Phi(\tau=2 \pi R)=\tilde{\phi}_{-}} D \Phi e^{-I_{0}[\Phi]} \tilde{\Psi}\left(\tau=\pi R+\tau_{0}\right) \tilde{\Psi}\left(\tau=\pi R-\tau_{0}\right),
\end{aligned}
$$

for $0 \leq \tau_{0} \leq \pi R$. In this equation, $\tilde{\Psi}=U \Psi U^{\dagger}$ is the image of $\Psi$ under conformal transformation and $\tilde{\Psi}\left(\tau_{*}\right)$ is the smearing of the local operator $\Psi(\tau, y)$ around a small neighborhood of $\tau=\tau_{*}$ and $y_{i}=0$ where $0<\tau<2 \pi R$ and $y_{i}$ are the coordinates on $S^{1}$ and $\mathbb{H}^{d-1}$ respectively. A pictorial representation of the path integral in eq. (3.21) is presented in figure 1. As we discuss in appendix B, the path integral in eq. (3.21) can be written in operator language. This yields the following expression for states $\rho$ and $\tilde{\rho}$

$$
U \rho U^{\dagger}=\tilde{\rho}=\frac{\tilde{\sigma} \tilde{\Psi}\left(\pi R+\tau_{0}\right) \tilde{\Psi}\left(\pi R-\tau_{0}\right)}{\left\langle\tilde{\Psi}\left(\pi R+\tau_{0}\right) \tilde{\Psi}\left(\pi R-\tau_{0}\right)\right\rangle_{\mathcal{H}^{\prime}}},
$$

where $\langle\ldots\rangle_{\mathcal{H}^{\prime}}$ is the correlation functions on $\mathcal{H}^{\prime}$.

Now consider the sandwiched state, $\rho_{(n)}$, defined in eq. (3.12). Note that we can write the sandwiched state, up to a normalization constant, as $\rho_{(n)} \sim \hat{\rho}_{(n)}^{n}$, where

$$
\hat{\rho}_{(n)}=\sigma^{\frac{1-n}{2 n}} \rho \sigma^{\frac{1-n}{2 n}} .
$$


Inverting this equation yields $\rho=\sigma^{\frac{n-1}{2 n}} \hat{\rho}_{(n)} \sigma^{\frac{n-1}{2 n}}$. As discussed in [2, 28, 55], this expression for $\rho$ allows us to write a path integral representation of $\hat{\rho}_{(n)}$. This follows from the observation that for $1<n<\frac{\pi R}{\tau_{0}},{ }^{4}$ the path integral representation of the matrix element in eq. (3.21) can be can be cut in $S^{1}$ direction as shown in figure 1. This yields that $\hat{\rho}_{(n)}$ can be written as a path integral (with two operator insertions) over $S^{1} \times \mathbb{H}^{d-1}$ where the radius of $S^{1}$ is now $R / n$. Now since $\rho_{(n)} \sim \hat{\rho}_{(n)}^{n}$, we take $n$ copies of $\hat{\rho}_{(n)}$ and glue them together. As a result, we find that, for integer $n \geq 1$, the sandwiched state $\rho_{(n)}$ can be written as a path integral over $\mathcal{H}^{\prime}$ with $2 n$ operators insertions $[2,28,55]$, as shown in figure 1.

In this paper, one of our goals is to use eq. (3.19) to study the refined Rényi relative entropy when the state $\rho$ is perturbatively expanded around the state $\sigma$ as in eq. (3.14). To write the state $\rho$ as in eq. (3.14), we take the operator $\Psi$ as a perturbation around the identity and write it as

$$
\Psi=1+\epsilon \mathcal{O}+O\left(\epsilon^{2}\right)
$$

Inserting this in eq. (3.22) and comparing it with eq. (3.14), we find

$$
U \rho^{(1)} U^{\dagger}=\tilde{\rho}^{(1)}=\tilde{\sigma} \tilde{\mathcal{O}}\left(\pi R+\tau_{0}\right)+\tilde{\sigma} \tilde{\mathcal{O}}\left(\pi R-\tau_{0}\right) .
$$

Since $\tilde{\mathcal{O}}$ are smeared operators, we can equivalently write eq. (3.25) as an integral over $\mathcal{H}^{\prime}$,

$$
U \rho^{(1)} U^{\dagger}=\tilde{\rho}^{(1)}=\int_{0}^{2 \pi R} d \tau \int_{H^{d-1}} d^{d-1} y \tilde{\lambda}(\tau, y) \tilde{\sigma} \tilde{\mathcal{O}}(\tau, y)
$$

where $\tilde{\lambda}$ is equal to the smearing function inside small neighborhoods of $\tau=\pi R \pm \tau_{0}$ and $y_{i}=0$ and vanishes everywhere outside these two neighborhoods. Also note that, by construction, the function $\tilde{\lambda}$ is symmetric around $\tau=\pi R$.

This finishes our discussion of the path integral construction of sandwiched state, $\rho_{(n)}$. In the next two sections, we will study the refined Rényi relative entropy between these state $\rho$ given in eq. (3.22) and state $\sigma$ in eq. (3.20).

\section{Perturbative refined Rényi relative entropy in a general CFT}

The perturbative calculation of relative entropy between the vacuum state and a slightly perturbed state of a CFT, both reduced to a spherical region, has been well-studied [1, 26, 27]. It was shown in [1] that the relative entropy at the lowest order in the perturbation parameter can be written as the symplectic flux of a scalar field through a Cauchy slice of an auxiliary AdS spacetime, as given in eq. (1.2). Note that this result was derived purely from CFT calculations without assuming the AdS/CFT correspondence. In fact, this result is true for all CFT's. In this sense, this result is a generalization of [26] where the same result was derived for holographic CFT's using the AdS/CFT correspondence.

\footnotetext{
${ }^{4}$ We can follow [2] and take the limit $\tau_{0} \rightarrow 0$ or we can follow [57] and take $R \rightarrow 0$. This will allow us to use the following construction for arbitrarily large $n$.
} 
Our goal in this section is to study the refined Rényi relative entropy between states of a general CFT reduced to a spherical region $B$ and, for simplicity, we take the radius of $B$ to be $R=1$. We take state $\sigma$ to be the reduced vacuum state as in eq. (3.20) and $\rho$ to be the perturbation around $\sigma$ as in eq. (3.14) and eq. (3.26). In the following, we show that refined Rényi relative entropy at lowest order in the perturbation parameter can also be written as the symplectic flux of some scalar field through a Cauchy slice of the aforementioned auxiliary AdS-spacetime. Hence, our result can be considered to be a one-parameter generalization of the result of [1] given in eq. (1.2).

As we discussed in section 3.2, the states $\sigma$ and $\rho$ are related to the states on the hyperbolic space by a unitary transformation. Using the invariance of the refined Rényi relative entropy under unitary transformations, we write

$$
\widetilde{S}_{n}(\rho \| \sigma)=\widetilde{S}_{n}\left(U^{\dagger} \tilde{\rho} U \| U^{\dagger} \tilde{\sigma} U\right)=\widetilde{S}_{n}(\tilde{\rho} \| \tilde{\sigma}),
$$

where $\tilde{\sigma}$ and $\tilde{\rho}$ are states on the hyperbolic space and their explicit forms are given in eq. (3.20) and eq. (3.22) respectively.

Now to compute the perturbative refined Rényi relative entropy, we use the general formula derived in eq. (3.19). By inserting the perturbation of $\tilde{\rho}$ given in eq. (3.26) in the general formula, we get ${ }^{5}$

$$
\begin{aligned}
\widetilde{S}_{n}^{(2)}(\rho \| \sigma)= & -\sum_{k=0}^{n-1} \sum_{j=0}^{n-1}\left(\prod_{i=1}^{2} \int_{0}^{2 \pi} d \tau_{i} \int_{H^{d-1}} d^{d-1} y_{i} \tilde{\lambda}\left(\tau_{i}, y_{i}\right)\right) \int_{-\infty}^{\infty} \frac{d s}{4 \sinh ^{2}\left(\frac{s+i \delta}{2}\right)} \\
& \times \operatorname{tr}\left(\tilde{\sigma}^{-\frac{i s}{2 \pi}+\frac{(k-j)}{n}} \tilde{\mathcal{O}}\left(\tau_{1}, y_{1}\right) \tilde{\sigma}^{1+\frac{i s}{2 \pi}-\frac{(k-j)}{n}} \tilde{\mathcal{O}}\left(\tau_{2}, y_{2}\right)\right)
\end{aligned}
$$

where $\widetilde{S}_{n}^{(2)}(\rho \| \sigma)$ is the refined Rényi relative entropy at the second order in the perturbation parameter as defined in eq. (3.18). Let us now try to simplify the above expression. We start by using eq. (3.20) and the Euclidean evolution

$$
\tilde{\sigma}^{a} \tilde{\mathcal{O}}(\tau, y) \tilde{\sigma}^{-a}=e^{-2 \pi a H} \tilde{\mathcal{O}}(\tau, y) e^{2 \pi a H}=\tilde{\mathcal{O}}(\tau-2 \pi a, y),
$$

to write eq. (4.2) as

$$
\begin{aligned}
\widetilde{S}_{n}^{(2)}(\rho \| \sigma)= & -\sum_{k=0}^{n-1} \sum_{j=0}^{n-1}\left(\prod_{i=1}^{2} \int_{0}^{2 \pi} d \tau_{i} \int_{H^{d-1}} d^{d-1} y_{i} \tilde{\lambda}\left(\tau_{i}, y_{i}\right)\right) \int_{-\infty}^{\infty} \frac{d s}{4 \sinh ^{2}\left(\frac{s+i \delta}{2}\right)} \\
& \times \operatorname{tr}\left(\tilde{\sigma} \tilde{\mathcal{O}}\left(\tau_{2}+2 \pi k / n, y_{2}\right) \tilde{\mathcal{O}}\left(\tau_{1}+2 \pi j / n+i s, y_{1}\right)\right) .
\end{aligned}
$$

Now we write the above equation in terms of a time-ordered correlation function on $\mathcal{H}^{\prime}=$ $S^{1} \times \mathbb{H}^{d-1}$ as [1]

$$
\begin{aligned}
\widetilde{S}_{n}^{(2)}(\rho \| \sigma)= & -\sum_{k=0}^{n-1} \sum_{j=0}^{n-1}\left(\prod_{i=1}^{2} \int_{0}^{2 \pi} d \tau_{i} \int_{H^{d-1}} d^{d-1} y_{i} \tilde{\lambda}\left(\tau_{i}, y_{i}\right)\right) \int_{-\infty}^{\infty} \frac{d s}{4 \sinh ^{2}\left(\frac{s+i \delta \operatorname{sgn}\left[\tau_{2 k}-\tau_{1 j}\right]}{2}\right)} \\
& \times\left\langle\mathcal{T} \tilde{\mathcal{O}}\left(\tau_{2}+2 \pi k / n, y_{2}\right) \tilde{\mathcal{O}}\left(\tau_{1}+2 \pi j / n+i s, y_{1}\right)\right\rangle_{\mathcal{H}^{\prime}}
\end{aligned}
$$

\footnotetext{
${ }^{5}$ Note that we have taken $R=1$ here.
} 


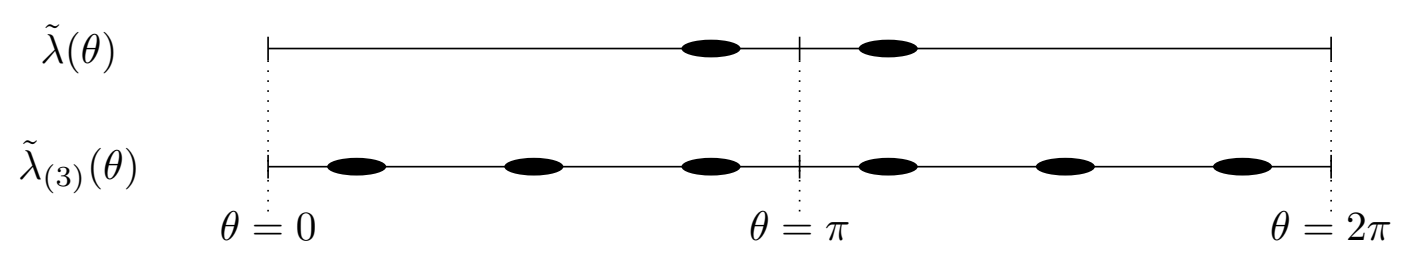

Figure 2. A pictorial representation of the source function $\tilde{\lambda}(\tau, y)$ and the $\mathbb{Z}_{3}$-symmetric source function $\tilde{\lambda}_{(3)}(\tau, y)$ where we have suppressed the $y$-coordinates for clarity. The functions are nonzero everywhere except in the shaded regions. Both the source function $\tilde{\lambda}$ and the $\mathbb{Z}_{3}$-symmetric source function $\tilde{\lambda}_{(3)}$ are symmetric around $\tau=\pi$. Whereas the source function $\tilde{\lambda}$ is non-zero only in the neighborhood of two points, the $\mathbb{Z}_{3}$-symmetric source function $\tilde{\lambda}_{(3)}$ is non-zero in the neighborhood of six points and has a periodicity of $2 \pi / 3$.

where $\tau_{2 k} \equiv\left(\tau_{2}+2 \pi k / n\right) \bmod 2 \pi$ and $\tau_{1 j}$ is defined similarly. Now by using the KMS condition [66-68] on the periodicity of the thermal two-point function

$$
\left\langle\mathcal{T} \tilde{\mathcal{O}}\left(\tau_{2}+2 \pi, y_{2}\right) \tilde{\mathcal{O}}\left(\tau_{1}, y_{1}\right)\right\rangle_{\mathcal{H}^{\prime}}=\left\langle\mathcal{T} \tilde{\mathcal{O}}\left(\tau_{2}, y_{2}\right) \tilde{\mathcal{O}}\left(\tau_{1}, y_{1}\right)\right\rangle_{\mathcal{H}^{\prime}}
$$

and by redefining the angular coordinates, we simplify eq. (4.5) to get

$$
\begin{aligned}
\widetilde{S}_{n}^{(2)}(\rho \| \sigma)= & -\left(\prod_{i=1}^{2} \int_{0}^{2 \pi} d \tau_{i} \int_{H^{d-1}} d^{d-1} y_{i} \tilde{\lambda}_{(n)}\left(\tau_{i}, y_{i}\right)\right) \int_{-\infty}^{\infty} \frac{d s}{4 \sinh ^{2}\left(\frac{s+i \delta \operatorname{sgn}\left[\tau_{2}-\tau_{1}\right]}{2}\right)} \\
& \times\left\langle\mathcal{T} \tilde{\mathcal{O}}\left(\tau_{2}, y_{2}\right) \tilde{\mathcal{O}}\left(\tau_{1}+i s, y_{1}\right)\right\rangle_{\mathcal{H}^{\prime}}
\end{aligned}
$$

where we have absorbed all the $n$ dependence in a $\mathbb{Z}_{n}$-symmetric source function, which we define as $^{6}$

$$
\tilde{\lambda}_{(n)}(\tau, y) \equiv \sum_{k=0}^{n-1} \tilde{\lambda}(\tau-2 \pi k / n, y) .
$$

Recall from section 3.2 that the source function, $\tilde{\lambda}$, was only non-zero in the small neighborhood of two points. On the contrary, the $\mathbb{Z}_{n}$-symmetric source function, $\tilde{\lambda}_{(n)}$, has a periodicity of $2 \pi / n$ and is non-zero in the neighborhood of $2 n$ points. Just like the source function, $\tilde{\lambda}$, the $\mathbb{Z}_{n}$-symmetric source function, $\tilde{\lambda}_{n}$, is also symmetric around $\tau=\pi$. A pictorial representation of the $\mathbb{Z}_{n}$-symmetric source function, $\tilde{\lambda}_{n}$, is presented in figure 2 .

In the following, we use eq. (4.7) to write the perturbative refined Rényi relative entropy as the symplectic flux. Note that an expression similar to eq. (4.7) was found in [1] for the perturbative relative entropy. In fact, eq. (4.7) reduces to the expression of perturbative relative entropy in [1] if we replace $\tilde{\lambda}_{(n)}$ with $\tilde{\lambda}$. This should not be surprising given that the refined Rényi relative entropy approaches the relative entropy in the limit $n \rightarrow 1$ and all

\footnotetext{
${ }^{6}$ The function $\tilde{\lambda}(\tau, y)$ in eq. (3.26) was defined only in the domain $\tau=[0,2 \pi)$. We extend this function beyond this domain by demanding the periodicity condition: $\tilde{\lambda}(\tau+2 \pi, y)=\tilde{\lambda}(\tau, y)$.
} 
the $n$ dependence in eq. (4.7) is encoded in the $\mathbb{Z}_{n}$-symmetric source function, $\tilde{\lambda}_{(n)}$. This is a useful observation as it implies that we can write perturbative refined Rényi relative entropy in eq. (4.7) in terms of the symplectic flux by simply changing the boundary conditions of the scalar field in eq. (1.2) from $\tilde{\lambda}$ to $\tilde{\lambda}_{(n)}$. For the sake of completeness, we will now briefly sketch the argument of [1] and derive the expression of the perturbative refined Rényi relative entropy in terms of the symplectic flux in the following.

\subsection{Refined Rényi relative entropy as a symplectic flux}

Let us consider a scalar in a fixed AdS-Rindler wedge. Recall that the metric of the AdS-Rindler wedge can be written as

$$
d s^{2}=-\left(r^{2}-1\right) d t^{2}+\frac{d r^{2}}{r^{2}-1}+r^{2} d s_{\mathbb{H}^{d-1}}^{2}
$$

For a CFT with a holographic dual, the AdS-Rindler wedge is precisely the bulk region that is dual to the vacuum state reduced to a spherical region. In this section, however, we treat it as an auxiliary spacetime without assuming the AdS/CFT correspondence.

Note that, after a Wick rotation, the AdS-Rindler patch in eq. (4.9) becomes a Euclidean black hole with $\mathcal{H}^{\prime}=S^{1} \times \mathbb{H}^{d-1}$ as the asymptotic boundary. The real time solution of the Lorentzian scalar field equation can be written in terms of a bulk-to-boundary Euclidean propagator as $[69,70]$

$$
\phi(r, t, y)=\int_{0}^{2 \pi} d \tau^{\prime} \int_{\mathbb{H}^{d-1}} d^{d-1} y^{\prime} \phi_{0}\left(\tau^{\prime}, y^{\prime}\right) K_{E}\left(r, i t, y \mid \tau^{\prime}, y^{\prime}\right)
$$

where $\{\tau, y\}$ are the coordinates on $\mathcal{H}^{\prime}$ and the bulk point in the bulk-to-boundary Euclidean propagator, $K_{E}$, is analytically continued to real time. The asymptotic boundary condition at $r \rightarrow \infty$ of the field, after analytic continuation, is fixed by $\phi_{0}(\tau, y)$.

The main insight that was used in [1] to relate the perturbative relative entropy to symplectic flux was that the two point function on $\mathcal{H}^{\prime}$ can be written as the symplectic flux of the scalar field on a constant radial slice $\left(r=r_{0}\right)$ of the AdS-Rindler wedge. That is, $[1]$

$$
\begin{aligned}
\left\langle\mathcal{T} \tilde{\mathcal{O}}\left(\tau, y_{1}\right) \tilde{\mathcal{O}}\left(i s, y_{2}\right)\right\rangle_{\mathcal{H}^{\prime}} & \\
= & -\int_{-\infty}^{\infty} d t \int_{\mathbb{H}^{d-1}} d^{d-1} y \omega_{\phi}\left(K_{E}\left(r_{0}, i t, y \mid \tau, y_{1}\right), K_{R}\left(r_{0}, t, y \mid s, y_{2}\right)\right)
\end{aligned}
$$

where $K_{R}$ is the retarded bulk-to-boundary propagator which is related to the Euclidean bulk-to-boundary propagator according to

$$
K_{R}\left(r, t, y \mid s, y^{\prime}\right)=i \Theta(t-s) \lim _{\mu \rightarrow 0^{+}}\left[K_{E}\left(r, i t, y \mid i s-\mu, y^{\prime}\right)-K_{E}\left(r, i t, y \mid i s+\mu, y^{\prime}\right)\right]
$$


Now we insert eq. (4.11) in eq. (4.7) and write the perturbative refined Rényi relative entropy as

$$
\begin{aligned}
\widetilde{S}_{n}^{(2)}(\rho \| \sigma)= & \left(\prod_{i=1}^{2} \int_{0}^{2 \pi} d \tau_{i} \int_{H^{d-1}} d^{d-1} y_{i} \tilde{\lambda}_{(n)}\left(\tau_{i}, y_{i}\right)\right) \int_{-\infty}^{\infty} \frac{d s}{4 \sinh ^{2}\left(\frac{s+i \delta \operatorname{sgn}\left[\tau_{2}-\tau_{1}\right]}{2}\right)} \\
& \times \int_{-\infty}^{\infty} d t \int_{\mathbb{H}^{d-1}} d^{d-1} y \omega_{\phi}\left(K_{E}\left(r_{0}, i t, y \mid \tau_{2}-\tau_{1}, y_{2}\right), K_{R}\left(r_{0}, t, y \mid s, y_{1}\right)\right) .
\end{aligned}
$$

The integral over $s$ was computed in [1]. We use that result here and write the perturbed refined Rényi relative entropy as

$$
\widetilde{S}_{n}^{(2)}(\rho \| \sigma)=-2 \pi\left(\prod_{i=1}^{2} \int_{\mathcal{H}^{\prime}} d X_{i} \tilde{\lambda}_{(n)}\left(X_{i}\right)\right) \int_{\Sigma_{0}} \omega_{\phi}\left(K_{E}\left(r, 0, y \mid X_{2}\right), \partial_{t} K_{E}\left(r, 0, y \mid X_{1}\right)\right),
$$

where $\Sigma_{0}$ is the $t=0$ slice in the AdS-Rindler patch and where $X=\{\tau, y\}$ are the coordinates on $\mathcal{H}^{\prime}$. Since the symplectic current $\omega_{\phi}$ is bilinear, we write eq. (4.14) as

$$
\widetilde{S}_{n}^{(2)}(\rho \| \sigma)=\int_{\Sigma_{0}} \omega_{\phi}\left(\Phi(r, t, y), £_{\xi} \Phi(r, t, y)\right)
$$

where $\xi^{a}=-2 \pi\left(\partial_{t}\right)^{a}$ is a killing vector field of the AdS-Rindler wedge as in eq. (2.8) and where we have defined

$$
\Phi(r, t, y)=\int_{\mathcal{H}^{\prime}} d X \tilde{\lambda}_{(n)}(X) K_{E}(r, t, y \mid X)
$$

By comparing this with eq. (4.10), we deduce that $\Phi(r, t, y)$ is the solution of the scalar field equation in bulk and the boundary condition of its analytic continuation is fixed by the $\mathbb{Z}_{n}$-symmetric source function, $\tilde{\lambda}_{(n)}$, defined in eq. (4.8).

Eq. (4.15) is the main result of this section. It shows that for integer $n \geq 1$, the perturbative refined Rényi relative entropy between the states $\rho$ in eq. (3.22) and $\sigma$ in eq. (3.20) is related to the symplectic flux of a scalar field through a Cauchy slice of the AdS-Rindler spacetime. More interestingly, it shows that all the dependence in the Rényi parameter $n$ is encoded in the $\mathbb{Z}_{n}$-symmetric source function, $\tilde{\lambda}_{n}$, which provides the boundary conditions for the scalar field. Also note that we recover eq. (1.2) from eq. (4.15) by taking the limit $n \rightarrow 1$. Hence, our result is a one-parameter generalization of eq. (1.2).

\section{Refined Rényi relative entropy in a holographic CFT}

We now focus on holographic CFT's on $R^{1, d-1}$. The relative entropy between an arbitrary state and a vacuum state reduced to a spherical region was studied in [2], where it was shown that the holographic dual of the relative entropy between these states is equal to the difference between certain conserved charges in the bulk dual of these states, as stated in eq. (1.3). 
In this section, we are interested in the refined Rényi relative entropy between two states of a holographic CFT reduced to a spherical region $B$. In particular, we will study the refined Rényi relative entropy between the state $\rho$ in eq. (3.22) and the vacuum state, $\sigma$, given in eq. (3.20). Moreover, we take the radius of the region $B$ to be $R=1$. We will show that the refined Rényi relative entropy between these states satisfies a holographic relation similar to eq. (1.3). Our result will be a one-parameter generalization of the result of [2] given in eq. (1.3).

Note that we can simply deduce the holographic formula for refined Rényi relative entropy using eq. (3.11) and eq. (1.3). According to eq. (3.11), the refined Rényi relative entropy between $\rho$ and $\sigma$ is equal to the relative entropy between the sandwiched state, $\rho_{(n)}$, and $\sigma$. If the sandwiched state, $\rho_{(n)}$, has a semi-classical gravity dual and if there exits a vector field in the bulk spacetime that satisfies boundary conditions similar to those in eq. (2.6) and eq. (2.9), then eq. (1.3) implies that the relative entropy between $\rho_{(n)}$ and $\sigma$ is equal to difference of conserved charges in the bulk duals of $\rho_{(n)}$ and $\sigma$. Then, by eq. (3.11), this is precisely the holographic expression for the refined Rényi relative entropy between states $\rho$ and $\sigma$.

Motivated by the above observation, we now discuss the bulk dual of the sandwiched state $\rho_{(n)}$. First, recall that the bulk region corresponding to the vacuum state reduced to a spherical region $B$ is the AdS-Rindler wedge [56], which is described by the metric given in eq. (4.9). The codimension-2 bifurcation surface, given by $r=1$ for any finite $t$ in the metric eq. (4.9), corresponds to the extremal surface corresponding to the boundary region $B$. We denote this surface by $\tilde{B}_{\sigma}$. Under Wick rotation, the metric of the AdS-Rindler wedge in eq. (4.9) becomes

$$
d s^{2}=\left(r^{2}-1\right) d \tau^{2}+\frac{d r^{2}}{r^{2}-1}+r^{2} d s_{\mathbb{H}^{d-1}}^{2}
$$

where $0 \leq \tau<2 \pi$ is imposed to ensure that there is no canonical singularity. This is the metric of the Euclidean black hole whose asymptotic boundary is $\mathcal{H}^{\prime}=S^{1} \times \mathbb{H}^{d-1}$. Note that $S^{1}$ contracts as we go deep into the bulk and it shrinks to zero size at a codimension-2 bifurcation surface, $\tilde{B}_{\sigma}$. This Euclidean geometry can be represented as a cigar geometry as shown in figure 3 .

Now recall from figure 1 that for integer $n \geq 1$, the sandwiched state $\rho_{(n)}$ can be prepared by a path integral over $\mathcal{H}^{\prime}$ with $2 n$ operator insertions. The Euclidean bulk dual of this state can be found by solving the Euclidean bulk equations of motions with appropriate boundary conditions. The boundary condition for the bulk Euclidean metric is that it approaches the metric of $\mathcal{H}^{\prime}$ near the asymptotic boundary. We also impose the condition that the Euclidean time, $\tau$, has a periodicity of $2 \pi$. Moreover, the sources for the operators inserted on the boundary provide the boundary conditions for the bulk matter fields according to the standard GKPW dictionary [69, 71]. This Euclidean geometry is shown in figure 3 .

Note that just like in the Euclidean black hole in eq. (5.1), the Euclidean circle in the Euclidean bulk dual of the sandwiched state contracts as we go into the bulk. We denote the codimension- 2 surface where the Euclidean circle shrinks to zero size by $\tilde{B}_{(n)}$. A general 

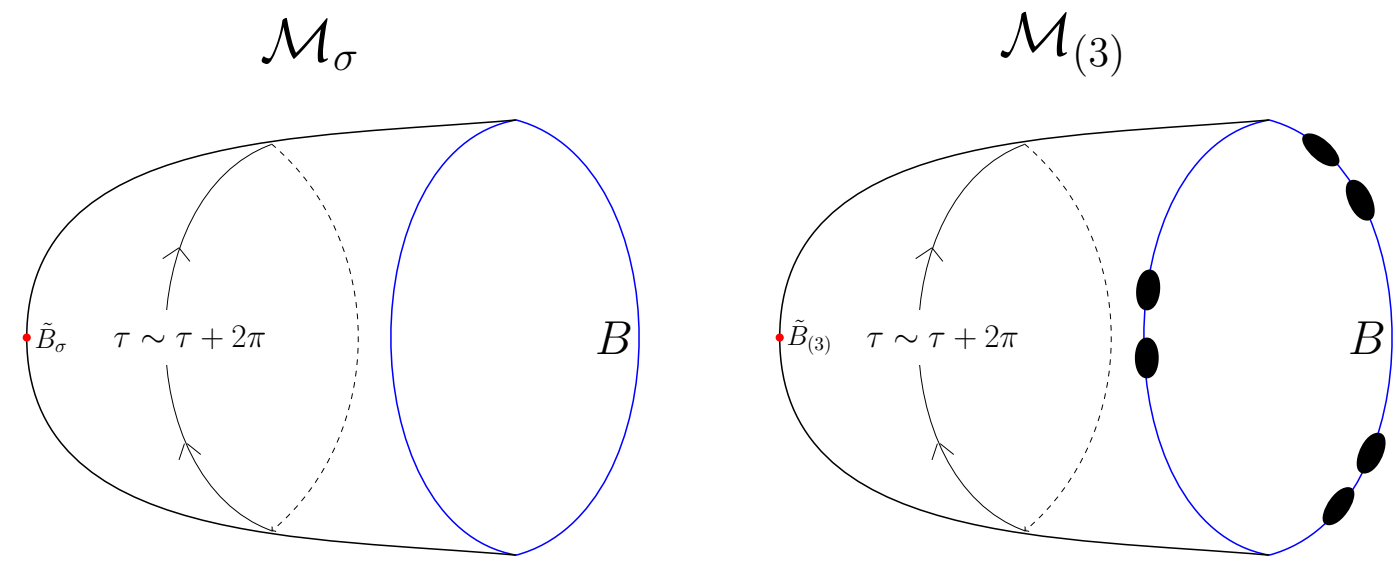

Figure 3. The Euclidean bulk geometries dual to the boundary states $\sigma$ (left) and $\rho_{(3)}$ (right). The boundary $B$ (shown in blue) is $S^{1} \times \mathbb{H}^{d-1}$. The Euclidean circle on the boundary contracts as one goes deep into the bulk and it is shrinks to zero size at a codimension-2 surface marked with a red dot. The shaded region on the boundary of $\mathcal{M}_{(3)}$ denotes the smeared operators inserted in the path integral representation of the sandwiched state, $\rho_{(3)}$; see figure 1 . The smearing function provides the $\mathbb{Z}_{3}$-symmetric boundary conditions for the bulk matter fields. (This figure is inspired by figure 2 of [2].)

ansatz for the Euclidean metric near $\tilde{B}_{(n)}$ can be written as [2]

$$
d s^{2}=\alpha_{(n)}^{2}(\tau, \hat{r}) d \tau^{2}+d \hat{r}^{2}+2 \beta_{i}^{(n)}(\tau, \hat{r}) d \tau d y^{i}+h_{i j} d y^{i} d y^{j},
$$

with $\alpha_{(n)}(\tau, \hat{r})=\hat{r}+O\left(\hat{r}^{2}\right)$ and $\beta_{i}^{(n)}(\tau, \hat{r})=O\left(\hat{r}^{2}\right)$, where $\hat{r}$ is the proper distance from $\tilde{B}_{(n)}$ and $y^{i}$ and $h_{i j}$ are coordinates and the induced metric on $\tilde{B}_{(n)}$.

Note that the Euclidean vector field $\xi_{E}^{a}=2 \pi\left(\partial_{\tau}\right)^{a}$ vanishes at $\tilde{B}_{(n)}$ and it satisfies

$$
\nabla^{a} \xi_{E}^{b}-\nabla^{b} \xi_{E}^{a}=4 \pi\left(\left(\partial_{\hat{r}}\right)^{a}\left(\partial_{\tau}\right)^{b}-\left(\partial_{\hat{r}}\right)^{b}\left(\partial_{\tau}\right)^{a}\right)
$$

as can be checked easily using the metric in eq. (5.2). Furthermore, the boundary condition that the Euclidean bulk metric approaches the metric of $\mathcal{H}^{\prime}$ near the asymptotic boundary guarantees that $\xi_{E}$ approaches the generator of the Euclidean time translation on the boundary. In other words, the vector field $\xi_{E}$ satisfies the Euclidean version of the boundary conditions in eq. (2.6) and eq. (2.9).

Having established that the sandwiched state (for integer $n \geq 1$ ) has a bulk dual, we now use eq. (1.3) to get

$$
S_{\text {rel }}\left(\rho_{(n)} \| \sigma\right)=H_{\xi}\left(\mathcal{M}_{(n)}\right)-H_{\xi}\left(\mathcal{M}_{\sigma}\right),
$$

where $\xi=i \xi_{E}=-2 \pi \partial_{t}$ as in eq. (2.8). Moreover, $\mathcal{M}_{\sigma}$ and $\mathcal{M}_{(n)}=\mathcal{M}_{\rho_{(n)}}$ denote the bulk spacetimes dual to the vacuum and the sandwiched state respectively. Now using eq. (3.11), we conclude that the refined Rényi relative entropy between states $\rho$ and $\sigma$ is given by

$$
\widetilde{S}_{n}(\rho \| \sigma)=H_{\xi}\left(\mathcal{M}_{(n)}\right)-H_{\xi}\left(\mathcal{M}_{\sigma}\right)
$$


Our result in eq. (5.5) needs to be contrasted with the holographic formula of the refined Rényi entropy which is defined in eq. (1.5). According to the holographic formula derived in [39], the refined Rényi entropy of a boundary state is equal to the area of a codimension-2 minimal area surface in some bulk spacetime other than the spacetime dual to the boundary state. In particular, the spacetime that one uses to compute refined Rényi entropy has to be constructed by introducing a cosmic brane and then accounting for its backreaction. Recently, the boundary state dual to this backreacted geometry has been identified in $[72,73]$. Our result in eq. (5.5) is similar in the sense that the refined Rényi relative entropy between states $\rho$ and $\sigma$ is not related to the conserved charge in the bulk dual of the state $\rho$. In fact, it is related to the conserved charge in the bulk dual of a sandwiched state, $\rho_{(n)}$.

Despite this similarity, there are two crucial differences between our holographic formula for the refined Rényi relative entropy and the holographic formula for refined Rényi entropy derived in [39]. One is that the holographic formula for the refined Rényi entropy is valid for a general boundary theory and for arbitrary boundary subregions. On the other hand, our results apply only if the boundary theory is a CFT and the boundary subregion is spherical. Secondly, the bulk geometry that one needs to compute the refined Rényi entropy has a conical singularity at the location of the cosmic brane [39], whereas the geometry that one needs for refined Rényi relative entropy, that is the bulk dual of the sandwiched state, is smooth. This is related to the fact that Rényi relative entropies, unlike Rényi entropies, are free of UV divergences. ${ }^{7}$

The holographic formula for refined Rényi relative entropy in eq. (5.5) is one of the main results of this paper. In the following, we discuss the implications of this result and its relation with the our perturbative result in section 4 .

\subsection{Positive energy theorems}

The result of [2] that the holographic dual of the relative entropy is related to the conserved charge as in eq. (1.3) led to interesting results. For example, the positive semi-definiteness of the relative entropy implies a positive energy theorem: the vacuum-subtracted conserved charge in the entanglement wedge of a spherical boundary region is non-negative. That is,

$$
H_{\xi}\left(\mathcal{M}_{\rho}\right)-H_{\xi}\left(\mathcal{M}_{\sigma}\right) \geq 0
$$

Note that eq. (5.6) is an infinite set of positivity conditions as it is true for all spherical boundary regions. We refer the interested readers to [2] for other gravitational constraints emerging from eq. (1.3).

Now we discuss a different positive energy theorem that arises from the holographic dual of refined Rényi relative entropy in eq. (5.5). Recall from eq. (3.9) that the refined Rényi relative entropy (for $n \geq 1$ ) cannot be smaller than the relative entropy. That is,

$$
\widetilde{S}_{n}(\rho \| \sigma) \geq S_{\text {rel }}(\rho \| \sigma) \quad \text { for } n \geq 1 .
$$

\footnotetext{
${ }^{7}$ We thank Tom Hartman for pointing this out.
} 
By combining this inequality with eq. (1.3) and eq. (5.5), we get

$$
H_{\xi}\left(\mathcal{M}_{(n)}\right) \geq H_{\xi}\left(\mathcal{M}_{(1)}\right) \quad \text { for } n \geq 1
$$

where $\mathcal{M}_{(1)}=\mathcal{M}_{\rho}$. Since the boundary conditions for the bulk dual of the sandwiched state are $\mathbb{Z}_{n}$-symmetric as shown in figure 3 , we conclude that making the boundary conditions symmetric in this manner cannot decrease the conserved charge in the entanglement wedge. Just like eq. (5.6), eq. (5.8) is also an infinite set of conditions as it is valid for any spherical boundary regions.

The positive energy condition in eq. (5.8) is interesting as it compares the conserved charges in two non-vacuum bulk geometries. In this sense, it is different from the usual positive energy theorems $[74,75]$ which compare the energy in a (non-vacuum) spacetime with the energy in a vacuum spacetime. Hence, we consider eq. (5.8) to be another nontrivial bulk consequence of a quantum information theoretic property.

It is known that the sandwiched Rényi relative entropy monotonically increases with the Rényi parameter $n$, as stated in eq. (3.5). It is therefore natural to ask if the refined Rényi relative entropy obeys monotonicity as well. We do not have a definitive answer to this question, though we present a simple example in appendix $\mathrm{C}$ in which the refined Rényi relative entropy increases monotonically with the Rényi parameter. If it can be shown that the refined Rényi relative entropy monotonically increases with the Rényi parameter either in general or for the set of states $\rho$ and $\sigma$ in eq. (3.22) and eq. (3.20) respectively, then this would imply a family of much stronger positive energy theorems than eq. (5.8). We leave this question to future work.

\subsection{Relation to the perturbative result}

As discussed earlier, the Euclidean bulk dual of the sandwiched state, $\rho_{(n)}$, is the solution of the bulk Euclidean equations of motions for the metric and the scalar field subject to $\mathbb{Z}_{n^{-}}$ symmetric boundary conditions. Here, we denote the boundary sources by $\tilde{\lambda}_{(n)}$ and assume that the boundary sources are "small," that is, we assume that the boundary sources can be written as $\tilde{\lambda}_{(n)}=\epsilon f_{(n)}$ where $\epsilon \ll 1$.

The boundary conditions for the bulk scalar field demand that the scalar field, $\Phi$, can be written as

$$
\Phi=\epsilon \Phi^{(1)}+O\left(\epsilon^{2}\right) .
$$

The bulk spacetime when $\epsilon=0$ is the (Euclidean) AdS-Rindler spacetime. The correction to the metric has to be computed by solving the gravitational equations of motions sourced by the stress-energy tensor of the bulk scalar field. Since the stress-energy tensor is quadratic in the scalar field, it goes like $O\left(\epsilon^{2}\right)$. Therefore, there is no backreaction at linear order in $\epsilon$ and hence, we can write the bulk metric as

$$
g=g^{(0)}+O\left(\epsilon^{2}\right),
$$

where $g^{(0)}$ is the metric of the (Euclidean) AdS-Rindler spacetime. Moreover, this implies that $\Phi^{(1)}$ is the solution of the scalar field on a fixed AdS-Rindler spacetime with $\mathbb{Z}_{n^{-}}$ symmetric boundary conditions. 
Now note that the conserved charge in the bulk dual of sandwiched state can be written as

$$
H_{\xi}[g]=H_{\xi}\left[g^{(0)}\right]+\epsilon H_{\xi}^{(1)}[g]+\epsilon^{2} H_{\xi}^{(2)}[g]+O\left(\epsilon^{3}\right),
$$

Since $\xi$ is the killing vector field of the AdS-Rindler spacetime, we know from eq. (2.26) that $H_{\xi}^{(1)}$ vanishes identically. Moreover, from eq. $(2.27)$, we know that $H_{\xi}^{(2)}$ is given by

$$
H_{\xi}^{(2)}=\int_{\Sigma_{0}} \omega_{\phi}\left(\Phi^{(1)}, £_{\xi} \Phi^{(1)}\right)
$$

where we have used the fact that there is no correction to the metric at linear order in $\epsilon$.

Now combining eq. (5.5) and eq. (5.12), we deduce that the refined Rényi relative entropy at the lowest order in the perturbation parameter is given by the symplectic flux of a scalar field, where the scalar field satisfies the $\mathbb{Z}_{n}$-symmetric boundary conditions. Hence, we recover eq. (4.15) from eq. (5.5). However, this should not be considered a derivation of eq. (4.15) as this analysis is only valid for CFT's with semi-classical holographic duals whereas eq. (4.15) is valid for all CFT's.

This finishes our discussion of the holographic dual of the refined Rényi relative entropy.

\section{Discussion}

In this paper, we have introduced a quantity related to the sandwiched Rényi relative entropy, which we called the 'refined' Rényi relative entropy, as defined in eq. (1.7). Like the sandwiched Rényi relative entropy, this quantity is a one-parameter generalization of relative entropy. In particular, we found that the refined Rényi relative entropy between two states can be written as the relative entropy of two related states, as in eq. (3.11). This identity relating the refined Rényi relative entropy with relative entropy played an important role in our analysis in this paper.

We derived a perturbative formula, eq. (3.19), for the refined Rényi relative entropy of two nearby states. Using this result, we studied the perturbative refined Rényi relative entropy when the reference state is the vacuum of a CFT reduced to a spherical region and the other state is a small perturbation thereof. We found that the perturbative refined Rényi relative entropy can be written as the symplectic flux of a scalar field through a Cauchy slice of the AdS-Rindler spacetime as in eq. (4.15).

We then studied the refined Rényi relative entropy for holographic CFT's. We argued that for a certain family of states reduced to a spherical region, the holographic dual of the refined Rényi relative entropy is related to certain conserved charges in the dual bulk spacetime. Combining this holographic result with an inequality in eq. (3.9) that the refined Rényi relative entropy must satisfy, we then proved certain 'positive-energy' theorems in the asymptotically locally AdS spacetimes.

We now discuss some possible directions in which the present work can be extended.

\section{Data processing inequality}

As discussed in section 3.1, the relative entropy and the sandwiched Rényi relative entropy do not increase under a CPTP map [44-48, 65]. This is known as the dataprocessing inequality, given in eq. (3.2). Since the partial trace operation is a CPTP 
map, the data-processing inequality implies that the relative and the sandwiched Rényi relative entropy for states of system $B$ is not greater than those for states of system $A$ if $B \subseteq A$, as stated in eq. (3.3).

A 'good' measure of the distinguishability of two quantum states must satisfy the property that it does not increase under a partial trace operation. This is because the partial trace could in general result in the loss of some potentially distinguishing information, thus rendering distinguishability of the states more difficult. It would be interesting to investigate if the refined Rényi relative entropy satisfies a dataprocessing inequality.

\section{Monotonicity in the Rényi parameter $n$}

Recall from eq. (3.5) that the sandwiched Rényi relative entropy monotonically increases with the Rényi parameter $n[45,47]$. We have derived a weaker version of this inequality for the refined Rényi relative entropy in eqs. (3.9)-(3.10). We have used eqs. (3.9)-(3.10) in section 5.1 to prove certain positive energy theorems in asymptotically locally AdS spacetimes.

It is not clear if the refined Rényi relative entropy monotonically increases with the Rényi parameter $n$ in general (although we showed it to be true in a simple example in appendix C). If this were true, then the holographic formula for the refined Rényi relative entropy in eq. (5.5) would lead to much stronger positive energy theorems than what we have found in this paper. Because of this, it would be worthwhile to explore if the refined Rényi relative entropy increases monotonically with $n$.

3. Generalization of quantum null energy condition (QNEC)

A QNEC is a local statement which puts a lower bound on the expectation value of the 'null-null' component of the stress-energy tensor in an arbitrary state $|\psi\rangle$ of a quantum field theory in $R^{1, d-1}[76]$. More precisely, it states that $\left\langle T_{k k}\right\rangle$ for any null vector $k$ at any point $p$ satisfies

$$
\left\langle T_{k k}\right\rangle \geq \frac{1}{2 \pi} S^{\prime \prime}(\rho),
$$

where $\rho$ is the state $|\psi\rangle$ reduced to any subregion $\Sigma_{p}$ such that the boundary of that subregion contains the point $p$. Moreover, $S(\rho)$ is the Von Neumann entropy of the state $\rho$ and prime denotes the infinitesimal variation of the subregion $\Sigma_{p}$ in the $k^{a}$ direction at point $p$.

In some specific cases, eq. (6.1) can be written in terms of the relative entropy. In particular, if the boundary of the entangling region lies on a null hypersurface, eq. (6.1) can be written as [11]

$$
S_{\text {rel }}^{\prime \prime}(\rho \| \sigma) \geq 0
$$

where $\sigma$ is the reduced vacuum state. This follows from known results about the modular Hamiltonian when the entangling surface lies on a null plane [77]. 
Recently, the sandwiched Rényi relative entropy between an excited state and a vacuum state of an arbitrary QFT reduced to a half-space was considered in [55]. It was found that the sandwiched Rényi relative entropy satisfies a bound analogous to eq. (6.2). It would be interesting to repeat this analysis for the refined Rényi relative entropy and see if it also satisfies such a bound.

\section{Refined Rényi relative entropy under $R G$ flow}

In this project, we have only studied the refined Rényi relative entropy between the states of a conformal field theory. It would be interesting to study it away from critical points. Specifically, one may deform a CFT by a relevant operator and study the refined Rényi relative entropy between the vacuum state of the deformed theory and that of the original CFT. This calculation, to lowest order in the coupling, can be done using the general perturbative formula in eq. (3.19). It would be fascinating if one can gain some insights on RG flow, similar to those in [78], by using inequalities in eqs. (3.9)-(3.10).

\section{Further Inequalities for the refined Rényi relative entropy through Holography}

It is also possible that further inequalities for the refined Rényi relative entropy can be discovered by exploiting holography. It would be interesting if the properties of the conserved charges in asymptotically locally AdS spacetimes can be used to find constraints obeyed by the refined Rényi relative entropy for holographic states that are not obeyed by that for all quantum states. These would be analogous to the constraints satisfied by the holographic entanglement entropy known as the holographic entropy cone $[79,80]$.

\section{Generalization of JLMS formula}

It is known that the relative entropy of two nearby boundary states is equal to the relative entropy of the corresponding bulk states [15]. This holographic relation was an important ingredient in proofs of bulk reconstruction [16-18]. It would be interesting to explore if an analogous relation holds for refined Rényi relative entropy and what its implications are for bulk reconstruction.

\section{Acknowledgments}

We would like to thank Nima Afkhami-Jeddi, Ahmed Almheiri, Tom Faulkner, Tom Hartman, Nima Lashkari, and Pratik Rath for useful discussions during the course of this work. NB is supported by the National Science Foundation under grant number 82248-1306744-PHPXH, by the Department of Energy under grant number DE-SC0019380, and by New York State Urban Development Corporation - Empire State Development - contract no. AA289. MM is supported by the US Department of Energy under grant number DE-SC0014123. 


\section{A Refined Rényi relative entropy as relative entropy}

In section 3.1, we claimed that the refined Rényi relative entropy between two states can be written as the relative entropy between two related states as in eq. (3.11). We found this identity very useful in deriving a general formula for the perturbative refined Rényi relative entropy, given in eq. (3.19). We applied this general perturbative formula in section 4 to show that the refined Rényi relative entropy between two states of any CFT reduced to a spherical region can be written as the symplectic flux of a scalar field through a Cauchy slice of the AdS-Rindler wedge as in eq. (4.15). Moreover, we combined the identity in eq. (3.11) with eq. (1.3) in section 5 to deduce the holographic dual of the refined Rényi relative entropy.

In this appendix, we present a derivation of the eq. (3.11). We start with the definition of refined Rényi relative entropy

$$
\widetilde{S}_{n}(\rho \| \sigma) \equiv n^{2} \partial_{n}\left(\frac{n-1}{n} S_{n}(\rho \| \sigma)\right)
$$

where

$$
S_{n}(\rho \| \sigma) \equiv \frac{1}{n-1} \log \operatorname{tr}\left\{\left(\sigma^{\frac{1-n}{2 n}} \rho \sigma^{\frac{1-n}{2 n}}\right)^{n}\right\},
$$

is the sandwiched Rényi relative entropy. Combining the above two expressions, we get

$$
\widetilde{S}_{n}(\rho \| \sigma) \equiv n^{2} \partial_{n}\left(\frac{1}{n} \log \operatorname{tr} \hat{\rho}_{(n)}^{n}\right),
$$

where we have defined a Hermitian matrix

$$
\hat{\rho}_{(n)} \equiv \sigma^{\frac{1-n}{n}} \rho \sigma^{\frac{1-n}{n}} .
$$

Taking the derivative w.r.t. $n$ in eq. (A.3), we get

$$
\widetilde{S}_{n}(\rho \| \sigma)=-\log \operatorname{tr} \hat{\rho}_{(n)}^{n}+\frac{n}{\operatorname{tr} \hat{\rho}_{(n)}^{n}} \partial_{n} \operatorname{tr} \hat{\rho}_{(n)}^{n} .
$$

Now using

$$
\partial_{n} \operatorname{tr} \hat{\rho}_{(n)}^{n}=\operatorname{tr}\left(\hat{\rho}_{(n)}^{n} \cdot \log \hat{\rho}_{(n)}\right)+n \operatorname{tr}\left(\hat{\rho}_{(n)}^{n-1} \cdot \partial_{n} \hat{\rho}_{(n)}\right)
$$

and

$$
\partial_{n} \hat{\rho}_{(n)}=-\frac{1}{2 n^{2}}\left(\log \sigma \cdot \hat{\rho}_{(n)}+\hat{\rho}_{(n)} \cdot \log \sigma\right)
$$

we write eq. (A.5) as

$$
\widetilde{S}_{n}(\rho \| \sigma)=-\log \operatorname{tr} \hat{\rho}_{(n)}^{n}+\frac{n}{\operatorname{tr} \hat{\rho}_{(n)}^{n}} \operatorname{tr}\left(\hat{\rho}_{(n)}^{n} \cdot \log \hat{\rho}_{(n)}\right)-\frac{1}{\operatorname{tr} \hat{\rho}_{(n)}^{n}} \operatorname{tr}\left(\hat{\rho}_{(n)}^{n} \cdot \log \sigma\right) .
$$

We simplify this result and write it as

$$
\widetilde{S}_{n}(\rho \| \sigma)=\operatorname{tr}\left(\rho_{(n)} \log \rho_{(n)}\right)-\operatorname{tr}\left(\rho_{(n)} \log \sigma\right),
$$


where

$$
\rho_{(n)} \equiv \frac{\hat{\rho}_{(n)}^{n}}{\operatorname{tr} \hat{\rho}_{(n)}^{n}}
$$

is precisely the sandwiched state defined in eq. (3.12). Now the expression in the r.h.s. of eq. (A.9) is the same as the definition of the relative entropy given in eq. (3.1). However, to relate the r.h.s. of eq. (A.9) with the relative entropy, we first need to show that the sandwiched state $\rho_{(n)}$ is a valid denisty matrix. In other words, it is a positive semi-definite matrix with unit trace. Note that, by construction, $\rho_{(n)}$ has unit trace. Moreover, the fact that $\rho_{(n)}$ is positive semi-definite follows from the fact that the matrix $\hat{\rho}_{(n)}$ in eq. (A.4) is positive semi-definite. To see this, note that the expectation value of $\hat{\rho}_{(n)}$ in any arbitrary state $|\psi\rangle$ is non-negative:

$$
\left\langle\psi\left|\hat{\rho}_{(n)}\right| \psi\right\rangle=\left\langle\psi^{\prime}|\rho| \psi^{\prime}\right\rangle \geq 0
$$

where $\left|\psi^{\prime}\right\rangle \equiv \sigma^{\frac{1-n}{n}}|\psi\rangle$. Since, the sandwiched state is positive semi-definite matrix with unit trace, it is a valid density matrix. Hence, we can write the r.h.s. of eq. (A.9) as relative entropy. More precisely,

$$
\widetilde{S}_{n}(\rho \| \sigma)=S_{\text {rel }}\left(\rho_{(n)} \| \sigma\right) .
$$

This finishes our derivation of eq. (3.11).

\section{B Conformal transformation and state $\rho$}

In this paper, we considered states that are obtained by acting on the vacuum of a CFT with a single smeared operator. In section 3.2, we stated that these states reduced to a spherical region are related to some states on the hyperbolic space by a unitary transformation. We claimed that the precise form of these reduced states is given in eq. (3.22). In this appendix, we present a derivation of eq. (3.22).

Consider a CFT with Euclidean action $I_{0}$ on $R^{d}$. We work in the coordinate systems in which the metric of $R^{d}$ is

$$
d s^{2}=d t_{E}^{2}+d r^{2}+r^{2} d \chi_{d-2}^{2}
$$

where $d \chi_{d-2}^{2}$ is the metric of a $(d-2)$-dimensional sphere of unit radius. Now consider a state $\left|\Psi\left(-t_{E 0}\right)\right\rangle \equiv \Psi\left(-t_{E 0}\right)|\Omega\rangle$, where $|\Omega\rangle$ is the vacuum state of the CFT and $\Psi\left(-t_{E 0}\right)$ is a local operator $\Psi(x)$ smeared in a small neighborhood around $t_{E}=-t_{E 0}<0$. Note that due to translation symmetry, this state is independent of where we insert the operator $\Psi$ at $t_{E}=-t_{E 0}$ slice. This means we can take the operator to be smeared in a small region around $\left(t_{E}, r\right)=\left(-t_{E 0}, 0\right)$. For simplicity, we assume that the operator $\Psi$ is Hermitian.

Now let $\rho$ be the state $\left|\Psi\left(-t_{E 0}\right)\right\rangle$ reduced to a spherical region $B$ of radius $R$. We take the region $B$ to be given by

$$
B: t_{E}=0 \text { and } \quad r \leq R .
$$


The density matrix of $\rho$ can can be written as a Euclidean path integral over $R^{d}$ with open cuts just above and below the region $B$ and with the insertion of $\Psi\left(-t_{E 0}\right)$ and $\Psi\left(t_{E 0}\right)$. That is, the matrix elements of $\rho$ (up to a normalization constant) are given by

$$
\left\langle\phi_{-}|\rho| \phi_{+}\right\rangle \sim \int_{\Phi\left(B_{ \pm}\right)=\phi_{ \pm}} D \Phi e^{-I_{0}[\Phi]} \Psi\left(-t_{E 0}\right) \Psi\left(t_{E 0}\right)
$$

where we have imposed the boundary conditions at the open cuts

$$
B_{ \pm}: \quad t_{E}=0^{ \pm} \quad \text { and } \quad r \leq R .
$$

Now note that $R^{d}$ can be conformally mapped to $\mathcal{H}^{\prime} \equiv S^{1} \times \mathbb{H}^{d-1}$. This conformal transformation is given by [30]

$$
t_{E}=R \frac{\sin (\tau / R)}{\cosh u+\cos (\tau / R)} \quad r=R \frac{\sinh u}{\cosh u+\cos (\tau / R)},
$$

where $0 \leq \tau \leq 2 \pi R$ and $0 \leq u<\infty$. Under this coordinate transformation, the metric of $R^{d}$ in eq. (B.1) becomes

$$
d s^{2}=(\cosh u+\cos (\tau / R))^{-2}\left(d \tau^{2}+R^{2}\left(d u^{2}+\sinh ^{2} u d \chi_{d-2}^{2}\right)\right)
$$

which is the same as the metric of $\mathcal{H}^{\prime}=S^{1} \times \mathbb{H}^{d-1}$, up to a conformal factor.

Using the conformal transformation in eq. (B.5), the matrix element in eq. (B.3) can be written as a path integral over $\mathcal{H}^{\prime}$. Note that the branch cut $B_{+}$maps to $\tau=0$ and $0 \leq u<\infty$ whereas the branch cut $B_{-}$maps to $\tau=2 \pi R$ and $0 \leq u<\infty$. Moreover, the points $\left(t_{E}= \pm t_{E 0}, r=0\right)$ map to $\left(\tau=\pi R \mp \tau_{0}, u=0\right)$, where

$$
\tau_{0}=2 R \arctan \left(R / t_{E 0}\right) .
$$

Hence, the matrix element of $\rho$ can be written as

$$
\left\langle\phi_{-}|\rho| \phi_{+}\right\rangle \sim \int_{\Phi(\tau=0)=\tilde{\phi}_{+}}^{\Phi(\tau=2 \pi R)=\tilde{\phi}_{-}} D \Phi e^{-I_{0}[\Phi]} \tilde{\Psi}\left(\tau=\pi R+\tau_{0}\right) \tilde{\Psi}\left(\tau=\pi R-\tau_{0}\right),
$$

where $|\tilde{\phi}\rangle \equiv U|\phi\rangle$ and $\tilde{\Psi} \equiv U \Psi U^{\dagger}$ are the unitary transformations of the states and operators under the aforementioned conformal transformation. This finishes our derivation of eq. (3.21).

Now note that we can write the path integral in eq. (B.8) in operator language. In the Schrodinger picture, we get

$$
\left\langle\phi_{-}|\rho| \phi_{+}\right\rangle \sim\left\langle\tilde{\phi}_{-}\left|e^{-\left(\pi R-\tau_{0}\right) H} \tilde{\Psi}(0) e^{-2 \tau_{0} H} \tilde{\Psi}(0) e^{-\left(\pi R-\tau_{0}\right) H}\right| \tilde{\phi}_{+}\right\rangle,
$$

whereas in the Heisenberg picture, we get

$$
\left\langle\phi_{-}|\rho| \phi_{+}\right\rangle \sim\left\langle\tilde{\phi}_{-}\left|e^{-2 \pi R H} \tilde{\Psi}\left(\pi R+\tau_{0}\right) \tilde{\Psi}\left(\pi R-\tau_{0}\right)\right| \tilde{\phi}_{+}\right\rangle,
$$


where $H$ is the Hamiltonian of the CFT on the hyperbolic space. Equivalently, we can write the above expression as

$$
U \rho U^{\dagger} \sim e^{-2 \pi R H} \tilde{\Psi}\left(\pi R+\tau_{0}\right) \tilde{\Psi}\left(\pi R-\tau_{0}\right) .
$$

In a simple case when the operator $\Psi$ is an identity operator, the state $\rho$ reduces to the vacuum state which we denote by $\sigma$. Therefore, from eq. (B.11), we deduce that the state $\sigma$ is

$$
U \sigma U^{\dagger} \sim e^{-2 \pi R H} \equiv \tilde{\sigma}
$$

which is the thermal state on the hyperbolic space, $\tilde{\sigma}$, up to a unitary transformation. Note that eq. (B.12) is the same as eq. (3.20) up to a normalization constant. Moreover, inserting eq. (B.12) in eq. (B.11) yields

$$
U \rho U^{\dagger} \sim \tilde{\sigma} \tilde{\Psi}\left(\pi R+\tau_{0}\right) \tilde{\Psi}\left(\pi R-\tau_{0}\right),
$$

which is the same as eq. (3.22) up to a normalization constant. This finishes the derivation of eq. (3.22) and our discussion of the states that we considered in this paper.

\section{Refined Rényi relative entropy for thermal states}

In this appendix, we study the refined Rényi relative entropy between two thermal states at different temperatures and show that it monotonically increases with the Rényi parameter $n$. Consider a quantum system with Hamiltonian, $H$, and two thermal states $\rho_{(0)}$ and $\rho_{(1)}$ such that

$$
\rho_{(i)}=\frac{e^{-\beta_{i} H}}{Z\left(\beta_{i}\right)} \quad \text { for } \quad i=\{1,2\}
$$

where

$$
Z(\beta) \equiv \operatorname{tr} e^{-\beta H}
$$

is the thermal partition function.

Now using eq. (3.11), the refined Rényi relative entropy between $\rho_{1}$ and $\rho_{0}$ is given by

$$
\widetilde{S}_{n}\left(\rho_{(1)} \| \rho_{(0)}\right)=S_{\text {rel }}\left(\rho_{(n)} \| \rho_{(0)}\right),
$$

where the sandwiched state, $\rho_{(n)}$, is

$$
\rho_{(n)}=\frac{\left(\rho_{(0)}^{\frac{1-n}{2 n}} \rho_{1} \rho_{(0)}^{\frac{1-n}{2 n}}\right)^{n}}{\operatorname{tr}\left(\rho_{(0)}^{\frac{1-n}{2 n}} \rho_{1} \rho_{(0)}^{\frac{1-n}{2 n}}\right)^{n}} .
$$

Since, $\rho_{(0)}$ and $\rho_{(1)}$ commute, we can simplify $\rho_{(n)}$ and write it as

$$
\rho_{(n)}=\frac{e^{-\beta_{n} H}}{Z\left(\beta_{n}\right)}
$$


where

$$
\beta_{n}=\beta_{0}+n\left(\beta_{1}-\beta_{0}\right) .
$$

We consider the case when $\beta_{1}>\frac{n-1}{n} \beta_{0}$. This ensures that $\beta_{n}>0$. Hence, we can think of $\rho_{(n)}$ as a thermal state with $n$ dependent temperature.

Now using the definition of the relative entropy in eq. (3.1), we write eq. (C.3) as

$$
\widetilde{S}_{n}\left(\rho_{(1)} \| \rho_{(0)}\right)=\operatorname{tr}\left(\rho_{(n)} \log \rho_{(n)}\right)+\beta_{0} \operatorname{tr}\left(\rho_{(n)} H\right)+\log Z\left(\beta_{0}\right),
$$

or equivalently as

$$
\widetilde{S}_{n}\left(\rho_{(1)} \| \rho_{(0)}\right)=\beta_{0} E\left(\beta_{n}\right)-S\left(\beta_{n}\right)+\log Z\left(\beta_{0}\right),
$$

where $E(\beta)$ and $S(\beta)$ are the average thermal energy and the thermal entropy of the system at temperature $1 / \beta$.

Next we take the derivative of eq. (C.8) w.r.t. $n$ and get

$$
\partial_{n} \widetilde{S}_{n}\left(\rho_{(1)} \| \rho_{(0)}\right)=\beta_{0} \partial_{n} E\left(\beta_{n}\right)-\partial_{n} S\left(\beta_{n}\right) .
$$

Now using the first law of thermodynamics, $\delta E(\beta)=\beta^{-1} \delta S(\beta)$, and using eq. (C.6), we simplify this to get

$$
\partial_{n} \widetilde{S}_{n}\left(\rho_{(1)}|| \rho_{(0)}\right)=-n\left(\beta_{1}-\beta_{0}\right) \partial_{n} E\left(\beta_{n}\right)=-\left.n\left(\beta_{1}-\beta_{0}\right)^{2}\left(\partial_{\beta} E(\beta)\right)\right|_{\beta=\beta_{n}} .
$$

Now note that $\partial_{\beta} E(\beta) \leq 0$. This can be seen as follows:

$$
\partial_{\beta} E(\beta)=\partial_{\beta}\langle H\rangle_{\beta}=\partial_{\beta} \frac{\operatorname{tr}\left(e^{-\beta H} H\right)}{\operatorname{tr}\left(e^{-\beta H}\right)}=-\left\langle H^{2}\right\rangle_{\beta}+\langle H\rangle_{\beta}^{2} \leq 0 .
$$

Combining this observation with eq. (C.10), we deduce that

$$
\partial_{n} \widetilde{S}_{n}\left(\rho_{(1)}|| \rho_{(0)}\right) \geq 0 .
$$

Hence, the refined Rényi relative entropy between two thermal states of the same system monotonically increases with the Rényi parameter.

Open Access. This article is distributed under the terms of the Creative Commons Attribution License (CC-BY 4.0), which permits any use, distribution and reproduction in any medium, provided the original author(s) and source are credited.

\section{References}

[1] T. Faulkner et al., Nonlinear gravity from entanglement in conformal field theories, JHEP 08 (2017) 057 [arXiv : 1705. 03026] [INSPIRE].

[2] N. Lashkari et al., Gravitational positive energy theorems from information inequalities, PTEP 12 (2016) 12C109 [arXiv:1605.01075]. 
[3] H. Casini, Relative entropy and the Bekenstein bound, Class. Quant. Grav. 25 (2008) 205021 [arXiv:0804.2182] [INSPIRE].

[4] R. Longo and F. Xu, Comment on the Bekenstein bound, J. Geom. Phys. 130 (2018) 113 [arXiv:1802.07184] [INSPIRE].

[5] A.C. Wall, A proof of the generalized second law for rapidly-evolving Rindler horizons, Phys. Rev. D 82 (2010) 124019 [arXiv: 1007.1493] [InSPIRE].

[6] A.C. Wall, Proof of the generalized second law for rapidly changing fields and arbitrary horizon slices, Phys. Rev. D 85 (2012) 104049 [Erratum ibid. D 87 (2013) 069904] [arXiv: 1105.3445].

[7] R. Bousso, H. Casini, Z. Fisher and J. Maldacena, Proof of a quantum bousso bound, Phys. Rev. D 90 (2014) 044002 [arXiv: 1404.5635] [INSPIRE].

[8] R. Bousso, H. Casini, Z. Fisher and J. Maldacena, Entropy on a null surface for interacting quantum field theories and the Bousso bound, Phys. Rev. D 91 (2015) 084030 [arXiv:1406.4545] [INSPIRE].

[9] T. Faulkner, R.G. Leigh, O. Parrikar and H. Wang, Modular Hamiltonians for deformed half-spaces and the averaged null energy condition, JHEP 09 (2016) 038.

[10] J. Koeller, S. Leichenauer, A. Levine and A. Shahbazi-Moghaddam, Local modular hamiltonians from the quantum null energy condition, Phys. Rev. D 97 (2018) 065011 [arXiv: 1702.00412] [INSPIRE].

[11] S. Leichenauer, A. Levine and A. Shahbazi-Moghaddam, Energy density from second shape variations of the von Neumann entropy, Phys. Rev. D 98 (2018) 086013 [arXiv:1802.02584] [INSPIRE].

[12] F. Ceyhan and T. Faulkner, Recovering the QNEC from the ANEC, arXiv:1812.04683 [INSPIRE].

[13] H. Casini, E. Teste and G. Torroba, Relative entropy and the RG flow, JHEP 03 (2017) 089 [arXiv: 1611.00016] [INSPIRE].

[14] N. Bao and H. Ooguri, Distinguishability of black hole microstates, Phys. Rev. D 96 (2017) 066017 [arXiv: 1705. 07943] [INSPIRE].

[15] D.L. Jafferis, A. Lewkowycz, J. Maldacena and S.J. Suh, Relative entropy equals bulk relative entropy, JHEP 06 (2016) 004 [arXiv: 1512.06431] [INSPIRE].

[16] X. Dong, D. Harlow and A.C. Wall, Reconstruction of bulk operators within the entanglement wedge in gauge-gravity duality, Phys. Rev. Lett. 117 (2016) 021601 [arXiv:1601.05416] [INSPIRE].

[17] T. Faulkner and A. Lewkowycz, Bulk locality from modular flow, JHEP 07 (2017) 151 [arXiv: 1704.05464] [INSPIRE].

[18] J. Cotler et al., Entanglement wedge reconstruction via universal recovery channels, Phys. Rev. X 9 (2019) 031011 [arXiv: 1704.05839] [INSPIRE].

[19] N. Lashkari, M.B. McDermott and M. Van Raamsdonk, Gravitational dynamics from entanglement 'thermodynamics', JHEP 04 (2014) 195 [arXiv:1308.3716] [INSPIRE].

[20] T. Faulkner et al., Gravitation from entanglement in holographic CFTs, JHEP 03 (2014) 051 [arXiv: 1312.7856] [INSPIRE].

[21] S. Banerjee et al., Constraining gravity using entanglement in AdS/CFT, JHEP 05 (2014) 029 [arXiv: 1401.5089] [INSPIRE]. 
[22] B. Swingle and M. Van Raamsdonk, Universality of gravity from entanglement, arXiv: 1405.2933 [INSPIRE].

[23] S. Banerjee, A. Kaviraj and A. Sinha, Nonlinear constraints on gravity from entanglement, Class. Quant. Grav. 32 (2015) 065006 [arXiv:1405.3743] [INSPIRE].

[24] J. Lin, M. Marcolli, H. Ooguri and B. Stoica, Locality of gravitational systems from entanglement of conformal field theories, Phys. Rev. Lett. 114 (2015) 221601 [arXiv: 1412.1879] [INSPIRE].

[25] N. Lashkari, C. Rabideau, P. Sabella-Garnier and M. Van Raamsdonk, Inviolable energy conditions from entanglement inequalities, JHEP 06 (2015) 067.

[26] N. Lashkari and M. Van Raamsdonk, Canonical energy is quantum Fisher information, JHEP 04 (2016) 153 [arXiv: 1508.00897] [INSPIRE].

[27] T. Faulkner, Bulk emergence and the RG flow of entanglement entropy, JHEP 05 (2015) 033 [arXiv: 1412.5648] [INSPIRE].

[28] N. Lashkari, Relative entropies in conformal field theory, Phys. Rev. Lett. 113 (2014) 051602 [arXiv: 1404.3216] [INSPIRE].

[29] D.D. Blanco, H. Casini, L.-Y. Hung and R.C. Myers, Relative entropy and holography, JHEP 08 (2013) 060 [arXiv: 1305.3182] [INSPIRE].

[30] V. Rosenhaus and M. Smolkin, Entanglement entropy: a perturbative calculation, JHEP 12 (2014) 179 [arXiv:1403.3733] [INSPIRE].

[31] V. Rosenhaus and M. Smolkin, Entanglement entropy, planar surfaces and spectral functions, JHEP 09 (2014) 119 [arXiv:1407.2891] [INSPIRE].

[32] A. Allais and M. Mezei, Some results on the shape dependence of entanglement and Rényi entropies, Phys. Rev. D 91 (2015) 046002 [arXiv: 1407.7249] [INSPIRE].

[33] A. Lewkowycz and E. Perlmutter, Universality in the geometric dependence of Renyi entropy, JHEP 01 (2015) 080 [arXiv: 1407.8171] [INSPIRE].

[34] V. Rosenhaus and M. Smolkin, Entanglement entropy for relevant and geometric perturbations, JHEP 02 (2015) 015 [arXiv: 1410.6530] [INSPIRE].

[35] M. Mezei, Entanglement entropy across a deformed sphere, Phys. Rev. D 91 (2015) 045038 [arXiv: 1411.7011] [INSPIRE].

[36] D. Carmi, On the shape dependence of entanglement entropy, JHEP 12 (2015) 043 [arXiv: 1506.07528] [INSPIRE].

[37] T. Faulkner, R.G. Leigh and O. Parrikar, Shape dependence of entanglement entropy in conformal field theories, JHEP 04 (2016) 088 [arXiv: 1511.05179] [INSPIRE].

[38] S. Leichenauer, M. Moosa and M. Smolkin, Dynamics of the area law of entanglement entropy, JHEP 09 (2016) 035 [arXiv: 1604.00388] [INSPIRE].

[39] X. Dong, The gravity dual of Renyi entropy, Nature Commun. 7 (2016) 12472 [arXiv: 1601.06788] [INSPIRE].

[40] S. Ryu and T. Takayanagi, Holographic derivation of entanglement entropy from AdS/CFT, Phys. Rev. Lett. 96 (2006) 181602 [hep-th/0603001] [INSPIRE].

[41] V.E. Hubeny, M. Rangamani and T. Takayanagi, A covariant holographic entanglement entropy proposal, JHEP 07 (2007) 062 [arXiv: 0705.0016] [INSPIRE].

[42] A. Lewkowycz and J. Maldacena, Generalized gravitational entropy, JHEP 08 (2013) 090 [arXiv: 1304.4926] [INSPIRE]. 
[43] X. Dong, A. Lewkowycz and M. Rangamani, Deriving covariant holographic entanglement, JHEP 11 (2016) 028 [arXiv: 1607.07506] [InSPIRE].

[44] M.M. Wilde, A. Winter and D. Yang, Strong converse for the classical capacity of entanglement-breaking and hadamard channels via a sandwiched renyi relative entropy, Commun. Math. Phys. 331 (2014) 593 [arXiv:1306.1586] [INSPIRE].

[45] M. Müller-Lennert et al., On quantum Rényi entropies: a new generalization and some properties, J. Math. Phys. 54 (2013) 122203 [arXiv:1306.3142].

[46] R.L. Frank and E.H. Lieb, Monotonicity of a relative Rényi entropy, J. Math. Phys. 54 (2013) 122201 [arXiv: 1306.5358].

[47] S. Beigi, Sandwiched Rényi divergence satisfies data processing inequality, J. Math. Phys. 54 (2013) 122202 [arXiv: 1306.5920].

[48] M. Mosonyi and T. Ogawa, Quantum hypothesis testing and the operational interpretation of the quantum Rényi relative entropies, Commun. Math. Phys. 334 (2015) 1617 [arXiv: 1309.3228].

[49] H. Araki, Type of von Neumann Algebra Associated with Free Field, Prog. Theor. Phys. 32 (1964) 956.

[50] R. Longo, Algebraic and modular structure of von Neumann algebras of physics, Commun. Math. Phys. 38 (1982) 551 [inSPIRE].

[51] K. Fredenhagen, On the modular structure of local algebras of observables, Comm. Math. Phys. 97 (1985) 79.

[52] H. Araki, Relative entropy of states of von Neumann algebras, Publ. Res. Inst. Math. Sci. 11 (1976) 809.

[53] H. Araki, Inequalities in von Neumann algebras, Les rencontres Physiciens-Mathématiciens de Strasbourg (RCP25) 22 (1975) 1.

[54] E. Witten, APS medal for exceptional achievement in research: invited article on entanglement properties of quantum field theory, Rev. Mod. Phys. 90 (2018) 045003 [arXiv: 1803. 04993] [INSPIRE].

[55] N. Lashkari, Constraining quantum fields using modular theory, JHEP 01 (2019) 059 [arXiv: 1810.09306] [INSPIRE].

[56] H. Casini, M. Huerta and R.C. Myers, Towards a derivation of holographic entanglement entropy, JHEP 05 (2011) 036 [arXiv: 1102.0440] [INSPIRE].

[57] T. Ugajin, Perturbative expansions of Rényi relative divergences and holography, arXiv: 1812.01135 [INSPIRE].

[58] A. Bernamonti, F. Galli, R.C. Myers and J. Oppenheim, Holographic second laws of black hole thermodynamics, JHEP 07 (2018) 111 [arXiv:1803.03633] [INSPIRE].

[59] A. May and E. Hijano, The holographic entropy zoo, JHEP 10 (2018) 036 [arXiv: 1806.06077] [INSPIRE].

[60] R.M. Wald, Black hole entropy is the Noether charge, Phys. Rev. D 48 (1993) R3427 [gr-qc/9307038] [INSPIRE].

[61] V. Iyer and R.M. Wald, Some properties of Noether charge and a proposal for dynamical black hole entropy, Phys. Rev. D 50 (1994) 846 [gr-qc/9403028] [InSPIRE].

[62] J. Lee and R.M. Wald, Local symmetries and constraints, J. Math. Phys. 31 (1990) 725 [INSPIRE]. 
[63] R.M. Wald and A. Zoupas, A general definition of 'conserved quantities' in general relativity and other theories of gravity, Phys. Rev. D 61 (2000) 084027 [gr-qc/9911095] [INSPIRE].

[64] S. Hollands and R.M. Wald, Stability of black holes and black branes, Commun. Math. Phys. 321 (2013) 629 [arXiv:1201.0463] [INSPIRE].

[65] G. Lindblad, Expectations and entropy inequalities for finite quantum systems, Comm. Math. Phys. 39 (1974) 111.

[66] R. Kubo, Statistical-mechanical theory of irreversible processes. I. General theory and simple applications to magnetic and conduction problems, J. Phys. Soc. Japan 12 (1957) 570.

[67] P.C. Martin and J.S. Schwinger, Theory of many particle systems. 1., Phys. Rev. 115 (1959) 1342 [INSPIRE].

[68] R. Haag, N.M. Hugenholtz and M. Winnink, On the equilibrium states in quantum statistical mechanics, Commun. Math. Phys. 5 (1967) 215 [INSPIRE].

[69] E. Witten, Anti-de Sitter space and holography, Adv. Theor. Math. Phys. 2 (1998) 253 [hep-th/9802150] [INSPIRE].

[70] D. Marolf et al., From Euclidean sources to lorentzian spacetimes in holographic conformal field theories, JHEP 06 (2018) 077 [arXiv: 1709.10101] [INSPIRE].

[71] S.S. Gubser, I.R. Klebanov and A.M. Polyakov, Gauge theory correlators from noncritical string theory, Phys. Lett. B 428 (1998) 105 [hep-th/9802109] [INSPIRE].

[72] C. Akers and P. Rath, Holographic Renyi entropy from quantum error correction, JHEP 05 (2019) 052 [arXiv: 1811.05171] [INSPIRE].

[73] X. Dong, D. Harlow and D. Marolf, Flat entanglement spectra in fixed-area states of quantum gravity, arXiv: 1811.05382 [INSPIRE].

[74] L.F. Abbott and S. Deser, Stability of gravity with a cosmological constant, Nucl. Phys. B 195 (1982) 76 [INSPIRE].

[75] E. Woolgar, The positivity of energy for asymptotically Anti-de Sitter space-times, Class. Quant. Grav. 11 (1994) 1881 [gr-qc/9404019] [INSPIRE].

[76] R. Bousso, Z. Fisher, S. Leichenauer and A.C. Wall, Quantum focusing conjecture, Phys. Rev. D 93 (2016) 064044 [arXiv: 1506. 02669] [INSPIRE].

[77] H. Casini, E. Teste and G. Torroba, Modular Hamiltonians on the null plane and the Markov property of the vacuum state, J. Phys. A 50 (2017) 364001 [arXiv:1703.10656] [InSPIRE].

[78] H. Casini, R. Medina, I. Salazar Landea and G. Torroba, Renyi relative entropies and renormalization group flows, JHEP 09 (2018) 166 [arXiv:1807.03305] [INSPIRE].

[79] P. Hayden, M. Headrick and A. Maloney, Holographic mutual information is monogamous, Phys. Rev. D 87 (2013) 046003 [arXiv:1107.2940] [INSPIRE].

[80] N. Bao et al., The holographic entropy cone, JHEP 09 (2015) 130 [arXiv:1505.07839] [INSPIRE]. 